REPRESENTATION THEORY

An Electronic Journal of the American Mathematical Society

Volume 12, Pages 186-207 (April 14, 2008)

S 1088-4165(08)00329-4

\title{
EXISTENCE OF KIRILLOV-RESHETIKHIN CRYSTALS FOR NONEXCEPTIONAL TYPES
}

\author{
MASATO OKADO AND ANNE SCHILLING
}

Dedicated to Professor Masaki Kashiwara on his sixtieth birthday

\begin{abstract}
Using the methods of Kang et al. and recent results on the characters of Kirillov-Reshetikhin modules by Nakajima and Hernandez, the existence of Kirillov-Reshetikhin crystals $B^{r, s}$ is established for all nonexceptional affine types. We also prove that the crystals $B^{r, s}$ of type $B_{n}^{(1)}, D_{n}^{(1)}$, and $A_{2 n-1}^{(2)}$ are isomorphic to recently constructed combinatorial crystals for $r$ not a spin node.
\end{abstract}

\section{INTRODUCTION}

The theory of crystal bases by Kashiwara 15 provides a remarkably powerful tool to study the representations of quantum algebras $U_{q}(\mathfrak{g})$. For instance, the calculation of tensor product multiplicities reduces to counting the number of crystal elements having certain properties. Although crystal bases are bases at $q=0$, one can "melt" them to get actual bases, called global crystal bases, for integrable highest weight representations of $U_{q}(\mathfrak{g})$. It turns out that the global crystal basis agrees with Lusztig's canonical basis [22, and it has many applications in representation theory.

The main focus of this paper is affine finite crystals, that is, crystal bases of finite-dimensional modules for quantum groups corresponding to affine Kac-Moody algebras $\mathfrak{g}$. These crystal bases were first developed by Kang et al. 13, 14, where it was also shown that integrable highest-weight $U_{q}(\mathfrak{g})$-modules of arbitrary level can be realized as semi-infinite tensor products of perfect crystals. This is known as the path realization. Many perfect crystals were proven to exist and explicitly constructed in 14 .

Irreducible finite-dimensional $U_{q}^{\prime}(\mathfrak{g})$-modules were classified by Chari and Pressley [4, 5] in terms of Drinfeld polynomials. It was conjectured by Hatayama et al. 7, 8, that a certain subset of such modules known as Kirillov-Reshetikhin (KR) modules $W_{s}^{(r)}$ have a crystal basis $B^{r, s}$. Here the index $r$ corresponds to a node of the Dynkin diagram of $\mathfrak{g}$ except the prescribed 0 and $s$ is an arbitrary positive integer. This conjecture was confirmed in many instances [2, 13, 14, 17, 19, 26, 34, but a proof for general $r$ and $s$ has not been available except type $A_{n}^{(1)}$ in [14]. Only recently the existence proof was completed in [27] for type $D_{n}^{(1)}$. Using the methods of [14] and recent results on the characters of KR modules [9, 10, 24, we

Received by the editors August 8, 2007 and, in revised form, February 26, 2008.

2000 Mathematics Subject Classification. Primary 17B37, 81R50; Secondary 05E15, 81R10.

(C)2008 American Mathematical Society 
establish the existence of Kirillov-Reshetikhin crystals $B^{r, s}$ for all nonexceptional affine types in this paper:

Theorem 1.1. The Kirillov-Reshetikhin module $W_{s}^{(r)}$ associated to any nonexceptional affine Kac-Moody algebra has a crystal basis $B^{r, s}$.

In addition we prove that for type $B_{n}^{(1)}, D_{n}^{(1)}$, and $A_{2 n-1}^{(2)}$ these crystals coincide with the combinatorial crystals of [30, 32. Throughout the paper we denote by $B^{r, s}$ the KR crystal associated with the KR module $W_{s}^{(r)}$. The combinatorial crystal of 30 is called $\tilde{B}^{r, s}$. Our second main result is the following theorem:

Theorem 1.2. For $1 \leq r \leq n-2$ for type $D_{n}^{(1)}, 1 \leq r \leq n-1$ for type $B_{n}^{(1)}$, $1 \leq r \leq n$ for type $A_{2 n-1}^{(2)}$ and $s \in \mathbb{Z}_{>0}$, the crystals $B^{r, s}$ and $\tilde{B}^{r, s}$ are isomorphic.

The key to the proof of Theorem 1.1 is Proposition 2.1 below, which is due to Kang et al. 14 and states that a finite-dimensional $U_{q}^{\prime}(\mathfrak{g})$-module having a prepolarization and certain $\mathbb{Z}$-form has a crystal basis if the dimensions of some particular weight spaces are not greater than the weight multiplicities of a fixed module and the values of the prepolarization of certain vectors in the module have some special properties. Using the fusion construction it is established that the KR modules have a prepolarization and $\mathbb{Z}$-form. The requirements on the dimensions follow from recent results by Nakajima [24] and Hernandez [9, 10. Necessary values of the prepolarization are calculated explicitly in Propositions 4.1, 4.4, and 4.6.

The isomorphism between the $\mathrm{KR}$ crystal $B^{r, s}$ and the combinatorial crystal $\tilde{B}^{r, s}$ is established by showing that isomorphisms as crystals with index sets $\{1,2,3, \ldots, n\}$ and $\{0,2,3, \ldots, n\}$ already uniquely determine the whole crystal.

Before presenting our results, let us offer some speculations on combinatorial realizations for the KR crystals. For type $A_{n}^{(1)}$ the crystals $B^{r, s}$ were constructed combinatorially by Shimozono 31 using the promotion operator. The promotion operator pr is the crystal analogue of the Dynkin diagram automorphism that maps node $i$ to node $i+1$ modulo $n+1$. The affine crystal operator $\tilde{f}_{0}$ is then given by $\tilde{f}_{0}=\operatorname{pr}^{-1} \circ \tilde{f}_{1} \circ$ pr. Similarly, the main tool used in 30] to construct the combinatorial crystals $\tilde{B}^{r, s}$ of type $B_{n}^{(1)}, D_{n}^{(1)}$, and $A_{2 n-1}^{(2)}$ is the crystal analogue of the Dynkin diagram automorphism that interchanges nodes 0 and 1. For type $C_{n}^{(1)}$ and $D_{n+1}^{(2)}$, there exists a Dynkin diagram automorphism $i \mapsto n-i$. It is our intention to exploit this symmetry to construct $\tilde{B}^{r, s}$ of type $C_{n}^{(1)}$ and $D_{n+1}^{(2)}$ explicitly in a future publication. For type $A_{2 n}^{(2)}$ no Dynkin diagram automorphism exists. However, it should still be possible to construct these crystals by looking at the $\{1,2, \ldots, n\}$ and $\{0,1,2, \ldots, n-1\}$ subcrystals as was done for $r=1$ in [14. Realizations of $B^{r, s}$ as virtual crystals were given in 28, 29.

The paper is organized as follows. In Section 2 we review necessary background on the quantum algebra $U_{q}^{\prime}(\mathfrak{g})$ and the fundamental representations. In particular, we review Proposition 2.1] of [14 which provides a criterion for the existence of a crystal pseudobase. In Section 3 we define KR modules by the fusion construction and show that these modules have a prepolarization. This reduces the existence proof for KR crystals to conditions stated in Proposition 3.7 These conditions are checked explicitly in Section 4 for the various types to prove Theorem 1.1. In Section 5 we review the combinatorial construction of the crystals $\tilde{B}^{r, s}$ of types 
$B_{n}^{(1)}, D_{n}^{(1)}$, and $A_{2 n-1}^{(2)}$ and prove in Section 6 that they are isomorphic to $B^{r, s}$, thereby establishing Theorem 1.2 .

\section{Quantum AFfine Algebra $U_{q}^{\prime}(\mathfrak{g})$ And Fundamental Representations}

2.1. Quantum affine algebra. Let $\mathfrak{g}$ be an affine Kac-Moody algebra and $U_{q}(\mathfrak{g})$ the quantum affine algebra associated to $\mathfrak{g}$. In this section $\mathfrak{g}$ can be any affine algebra. For the notation of $\mathfrak{g}$ or $U_{q}(\mathfrak{g})$ we follow [17. For instance, $P$ is the weight lattice, $I$ is the index set of simple roots, and $\left\{\alpha_{i}\right\}_{i \in I}$ (resp. $\left\{h_{i}\right\}_{i \in I}$ ) is the set of simple roots (resp. coroots). Let $($,$) be the inner product on P$ normalized by $(\delta, \lambda)=\langle c, \lambda\rangle$ for any $\lambda \in P$ as in 12, where $c$ is the canonical central element and $\delta$ is the generator of null roots. We choose a positive integer $d$ such that $\left(\alpha_{i}, \alpha_{i}\right) / 2 \in$ $\mathbb{Z} d^{-1}$ for any $i \in I$ and set $q_{s}=q^{1 / d}$. Then $U_{q}(\mathfrak{g})$ is the associative algebra over $\mathbb{Q}\left(q_{s}\right)$ with 1 generated by $e_{i}, f_{i}(i \in I), q^{h}\left(h \in d^{-1} P^{*}, P^{*}=\operatorname{Hom}_{\mathbb{Z}}(P, \mathbb{Z})\right)$ with certain relations. By convention, we set $q_{i}=q^{\left(\alpha_{i}, \alpha_{i}\right) / 2}, t_{i}=q_{i}^{h_{i}},[m]_{i}=\left(q_{i}^{m}-\right.$ $\left.q_{i}^{-m}\right) /\left(q_{i}-q_{i}^{-1}\right),[n]_{i} !=\prod_{m=1}^{n}[m]_{i}, e_{i}^{(n)}=e_{i}^{n} /[n]_{i} !, f_{i}^{(n)}=f_{i}^{n} /[n]_{i} !$.

Let $\left\{\Lambda_{i}\right\}_{i \in I}$ be the set of fundamental weights. Then we have $P=\bigoplus_{i} \mathbb{Z} \Lambda_{i} \oplus \mathbb{Z} \delta$. We set

$$
P_{c l}=P / \mathbb{Z} \delta .
$$

Similar to the quantum algebra $U_{q}(\mathfrak{g})$ which is associated with $P$, we can also consider $U_{q}^{\prime}(\mathfrak{g})$, which is associated with $P_{c l}$, namely, the subalgebra of $U_{q}(\mathfrak{g})$ generated by $e_{i}, f_{i}, q^{h}\left(h \in d^{-1}\left(P_{c l}\right)^{*}\right)$.

Next we introduce two subalgebras ("ZZZ-forms") $U_{q}(\mathfrak{g})_{K_{\mathbb{Z}}}$ and $U_{q}(\mathfrak{g})_{\mathbb{Z}}$ of $U_{q}(\mathfrak{g})$. Let $A$ be the subring of $\mathbb{Q}\left(q_{s}\right)$ consisting of rational functions without poles at $q_{s}=0$. We introduce the subalgebras $A_{\mathbb{Z}}$ and $K_{\mathbb{Z}}$ of $\mathbb{Q}\left(q_{s}\right)$ by

$$
\begin{aligned}
A_{\mathbb{Z}} & =\left\{f\left(q_{s}\right) / g\left(q_{s}\right) \mid f\left(q_{s}\right), g\left(q_{s}\right) \in \mathbb{Z}\left[q_{s}\right], g(0)=1\right\}, \\
K_{\mathbb{Z}} & =A_{\mathbb{Z}}\left[q_{s}^{-1}\right] .
\end{aligned}
$$

Then we have

$$
K_{\mathbb{Z}} \cap A=A_{\mathbb{Z}}, \quad A_{\mathbb{Z}} / q_{s} A_{\mathbb{Z}} \simeq \mathbb{Z} .
$$

We then define $U_{q}(\mathfrak{g})_{K_{\mathbb{Z}}}$ as the $K_{\mathbb{Z}}$-subalgebra of $U_{q}(\mathfrak{g})$ generated by $e_{i}, f_{i}, q^{h}(i \in$ $\left.I, h \in d^{-1} P^{*}\right) . \quad U_{q}(\mathfrak{g})_{\mathbb{Z}}$ is defined as the $\mathbb{Z}\left[q_{s}, q_{s}^{-1}\right]$-subalgebra of $U_{q}(\mathfrak{g})$ generated by $e_{i}^{(n)}, f_{i}^{(n)},\left\{\begin{array}{c}t_{i} \\ n\end{array}\right\}_{i}\left(i \in I, n \in \mathbb{Z}_{>0}\right)$ and $q^{h}\left(h \in d^{-1} P^{*}\right)$. Here we have set $\left\{\begin{array}{l}x \\ n\end{array}\right\}_{i}=$ $\prod_{k=1}^{n}\left(q_{i}^{1-k} x-q_{i}^{k-1} x^{-1}\right) /[n]_{i} ! . \quad U_{q}(\mathfrak{g})_{\mathbb{Z}}$ is a $\mathbb{Z}\left[q_{s}, q_{s}^{-1}\right]$-subalgebra of $U_{q}(\mathfrak{g})_{K_{\mathbb{Z}}}$. We can also introduce subalgebras $U_{q}^{\prime}(\mathfrak{g})_{K_{\mathbb{Z}}}$ and $U_{q}^{\prime}(\mathfrak{g})_{\mathbb{Z}}$ by replacing $q^{h}\left(h \in d^{-1} P^{*}\right)$ with $q^{h}\left(h \in d^{-1}\left(P_{c l}\right)^{*}\right)$ in the generators.

We define a total order on $\mathbb{Q}\left(q_{s}\right)$ by

$$
f>g \text { if and only if } f-g \in \bigsqcup_{n \in \mathbb{Z}}\left\{q_{s}^{n}\left(c+q_{s} A\right) \mid c>0\right\}
$$

and $f \geq g$ if $f>g$ or $f=g$.

Let $M$ and $N$ be $U_{q}(\mathfrak{g})\left(\right.$ or $\left.U_{q}^{\prime}(\mathfrak{g})\right)$-modules. A bilinear form $():, M \otimes_{\mathbb{Q}\left(q_{s}\right)} N \rightarrow$ $\mathbb{Q}\left(q_{s}\right)$ is called an admissible pairing if it satisfies

$$
\begin{aligned}
\left(q^{h} u, v\right) & =\left(u, q^{h} v\right), \\
\left(e_{i} u, v\right) & =\left(u, q_{i}^{-1} t_{i}^{-1} f_{i} v\right), \\
\left(f_{i} u, v\right) & =\left(u, q_{i}^{-1} t_{i} e_{i} v\right),
\end{aligned}
$$


for all $u \in M$ and $v \in N$. Equation (2.1) implies

$$
\left(e_{i}^{(n)} u, v\right)=\left(u, q_{i}^{-n^{2}} t_{i}^{-n} f_{i}^{(n)} v\right), \quad\left(f_{i}^{(n)} u, v\right)=\left(u, q_{i}^{-n^{2}} t_{i}^{n} e_{i}^{(n)} v\right) .
$$

A symmetric bilinear form (, ) on $M$ is called a prepolarization of $M$ if it satisfies (2.1) for $u, v \in M$. A prepolarization is called a polarization if it is positive definite with respective to the order on $\mathbb{Q}\left(q_{s}\right)$.

2.2. Criterion for the existence of a crystal pseudobase. Here we recall the criterion for the existence of a crystal pseudobase given in [14. We do not review the notion of crystal bases, but refer the reader to [15]. We only note that $q$ in the definition of crystal base in [15] should be replaced by $q_{s}$ according to the normalization of the inner product $($,$) on P$. We say $(L, B)$ is a crystal pseudobase of an integrable $U_{q}(\mathfrak{g})$ (or $U_{q}^{\prime}(\mathfrak{g})$ )-module $M$, if (i) $L$ is a crystal lattice of $M$, (ii) $B=B^{\prime} \sqcup\left(-B^{\prime}\right)$ where $B^{\prime}$ is a $\mathbb{Q}$-base of $L / q_{s} L$, (iii) $B=\bigsqcup_{\lambda \in P} B_{\lambda}$ where $B_{\lambda}=B \cap\left(L_{\lambda} / q_{s} L_{\lambda}\right)$, (iv) $\tilde{e}_{i} B \subset B \sqcup\{0\}, \tilde{f}_{i} B \subset B \sqcup\{0\}$, and (v) for $b, b^{\prime} \in B$, $b^{\prime}=\tilde{f}_{i} b$ if and only if $b=\tilde{e}_{i} b^{\prime}$. Note that only the condition (ii) is replaced from the definition of the crystal base.

Let $\mathfrak{g}_{0}$ be the finite-dimensional simple Lie algebra whose Dynkin diagram is obtained by removing the 0 -vertex from that of $\mathfrak{g}$. In this paper we specify the 0 -vertex as in 12 and set $I_{0}=I \backslash\{0\}$. Let $\bar{P}_{+}$be the set of dominant integral weights of $\mathfrak{g}_{0}$ and $\bar{V}(\lambda)$ be the irreducible highest weight $U_{q}\left(\mathfrak{g}_{0}\right)$-module of highest weight $\lambda$ for $\lambda \in \bar{P}_{+}$. The following proposition is easily obtained by combining Proposition 2.6.1 and 2.6.2 of [14].

Proposition 2.1. Let $M$ be a finite-dimensional integrable $U_{q}^{\prime}(\mathfrak{g})$-module. Let $(,$,$) be a prepolarization on M$, and $M_{K_{\mathbb{Z}}}$ a $U_{q}^{\prime}(\mathfrak{g})_{K_{\mathbb{Z}}}$-submodule of $M$ such that $\left(M_{K_{\mathbb{Z}}}, M_{K_{\mathbb{Z}}}\right) \subset K_{\mathbb{Z}}$. Let $\lambda_{1}, \ldots, \lambda_{m} \in \bar{P}_{+}$, and assume that the following conditions hold:

$$
\begin{aligned}
& \qquad \operatorname{dim} M_{\lambda_{k}} \leq \sum_{j=1}^{m} \operatorname{dim} \bar{V}\left(\lambda_{j}\right)_{\lambda_{k}} \text { for } k=1, \ldots, m . \\
& \text { There exist } u_{j} \in\left(M_{K_{\mathbb{Z}}}\right)_{\lambda_{j}}(j=1, \ldots, m) \text { such that }\left(u_{j}, u_{k}\right) \in \delta_{j k}+q_{s} A, \\
& \text { and }\left(e_{i} u_{j}, e_{i} u_{j}\right) \in q_{s} q_{i}^{-2\left(1+\left\langle h_{i}, \lambda_{j}\right\rangle\right)} \text { A for any } i \in I_{0} .
\end{aligned}
$$

Set $L=\{u \in M \mid(u, u) \in A\}$ and set $B=\left\{b \in M_{K_{\mathbb{Z}}} \cap L / M_{K_{\mathbb{Z}}} \cap q_{s} L \mid(b, b)_{0}=1\right\}$. Here $(,)_{0}$ is the $\mathbb{Q}$-valued symmetric bilinear form on $L / q_{s} L$ induced by $($,$) .$ Then we have the following:

(i) (, ) is a polarization on $M$.

(ii) $M \simeq \bigoplus_{j} \bar{V}\left(\lambda_{j}\right)$ as $U_{q}\left(\mathfrak{g}_{0}\right)$-modules.

(iii) $(L, B)$ is a crystal pseudobase of $M$.

2.3. Fundamental representations. For any $\lambda \in P$, Kashiwara defined a $U_{q}(\mathfrak{g})$ module $V(\lambda)$ called extremal weight module [16. We briefly recall its definition. Let $W$ be the Weyl group associated to $\mathfrak{g}$ and $s_{i}$ the simple reflection for $\alpha_{i}$. Let $M$ be an integrable $U_{q}(\mathfrak{g})$-module. A vector $u_{\lambda}$ of weight $\lambda \in P$ is called an extremal 
vector if there exists a set of vectors $\left\{u_{w \lambda}\right\}_{w \in W}$ satisfying

$$
\begin{aligned}
& u_{w \lambda}=u_{\lambda} \text { for } w=e, \\
& \text { if }\left\langle h_{i}, w \lambda\right\rangle \geq 0 \text {, then } e_{i} u_{w \lambda}=0 \text { and } f_{i}^{\left(\left\langle h_{i}, w \lambda\right\rangle\right)} u_{w \lambda}=u_{s_{i} w \lambda}, \\
& \text { if }\left\langle h_{i}, w \lambda\right\rangle \leq 0 \text {, then } f_{i} u_{w \lambda}=0 \text { and } e_{i}^{\left(-\left\langle h_{i}, w \lambda\right\rangle\right)} u_{w \lambda}=u_{s_{i} w \lambda} .
\end{aligned}
$$

Then $V(\lambda)$ is defined to be the $U_{q}(\mathfrak{g})$-module generated by $u_{\lambda}$ with the defining relations that $u_{\lambda}$ is an extremal vector. For our purpose, we only need $V(\lambda)$ when $\lambda=\varpi_{r}$ for $r \in I_{0}$, where $\varpi_{r}$ is a level 0 fundamental weight

$$
\varpi_{r}=\Lambda_{r}-\left\langle c, \Lambda_{r}\right\rangle \Lambda_{0}
$$

Then the following facts are known.

Proposition 2.2 ([17, Proposition 5.16]).

(i) $V\left(\varpi_{r}\right)$ is an irreducible integrable $U_{q}(\mathfrak{g})$-module.

(ii) $\operatorname{dim} V\left(\varpi_{r}\right)_{\mu}<\infty$ for any $\mu \in P$.

(iii) $\operatorname{dim} V\left(\varpi_{r}\right)_{\mu}=1$ for any $\mu \in W \varpi_{r}$.

(iv) wt $V\left(\varpi_{r}\right)$ is contained in the intersection of $\varpi_{r}+\sum_{i \in I} \mathbb{Z} \alpha_{i}$ and the convex hull of $W \varpi_{r}$.

(v) $V\left(\varpi_{r}\right)$ has a global crystal base $\left(L\left(\varpi_{r}\right), B\left(\varpi_{r}\right)\right)$.

(vi) Any integrable $U_{q}(\mathfrak{g})$-module generated by an extremal weight vector of weight $\varpi_{r}$ is isomorphic to $V\left(\varpi_{r}\right)$.

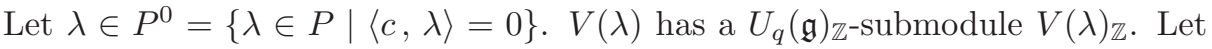
$\{G(b)\}_{b \in B(\lambda)}$ stand for the global base of $V(\lambda)$. The following result was shown in [33. for $\mathfrak{g}$ simply laced and $\lambda=\varpi_{r}$, in [23] for $\mathfrak{g}$ simply laced and $\lambda$ is arbitrary, and in [1] for $\mathfrak{g}$ and $\lambda$ arbitrary.

\section{Proposition 2.3.}

(i) There exists a prepolarization (, ) on $V(\lambda)$.

(ii) $\{G(b)\}_{b \in B(\lambda)}$ is almost orthonormal with respect to (, ), that is, $\left(G(b), G\left(b^{\prime}\right)\right)$ $\equiv \delta_{b b^{\prime}} \bmod q_{s} \mathbb{Z}\left[q_{s}\right]$.

Let $d_{r}$ be a positive integer such that

$$
\left\{k \in \mathbb{Z} \mid \varpi_{r}+k \delta \in W \varpi_{r}\right\}=\mathbb{Z} d_{r} .
$$

We note that $d_{r}=\max \left(1,\left(\alpha_{r}, \alpha_{r}\right) / 2\right)$ except in the case $d_{r}=1$ when $\mathfrak{g}=A_{2 n}^{(2)}$ and $r=n$. Then there exists a $U_{q}^{\prime}(\mathfrak{g})$-linear automorphism $z_{r}$ of $V\left(\varpi_{r}\right)$ of weight $d_{r} \delta$ sending $u_{\varpi_{r}}$ to $u_{\varpi_{r}+d_{r} \delta}$. Hence we can define a $U_{q}^{\prime}(\mathfrak{g})$-module $W\left(\varpi_{r}\right)$ by

$$
W\left(\varpi_{r}\right)=V\left(\varpi_{r}\right) /\left(z_{r}-1\right) V\left(\varpi_{r}\right)
$$

This module is called a fundamental representation.

For a $U_{q}^{\prime}(\mathfrak{g})$-module $M$ let $M_{\text {aff }}$ denote the $U_{q}^{\prime}(\mathfrak{g})$-module $\mathbb{Q}\left(q_{s}\right)\left[z, z^{-1}\right] \otimes M$ with the actions of $e_{i}$ and $f_{i}$ by $z^{\delta_{i 0}} \otimes e_{i}$ and $z^{-\delta_{i 0}} \otimes f_{i}$. For $a \in \mathbb{Q}\left(q_{s}\right)$ we define the $U_{q}^{\prime}(\mathfrak{g})$-module $M_{a}$ by $M_{\text {aff }} /(z-a) M_{\text {aff }}$.

Proposition 2.4 ([17, Proposition 5.17]).

(i) $W\left(\varpi_{r}\right)$ is a finite-dimensional irreducible integrable $U_{q}^{\prime}(\mathfrak{g})$-module.

(ii) For any $\mu \in$ wt $V\left(\varpi_{r}\right), W\left(\varpi_{r}\right)_{c l(\mu)} \simeq V\left(\varpi_{r}\right)_{\mu}$. Here the map cl stands for the canonical projection $P \longrightarrow P_{c l}$.

(iii) $\operatorname{dim} W\left(\varpi_{r}\right)_{c l(\mu)}=1$ for any $\mu \in W \varpi_{r}$. 
(iv) wt $W\left(\varpi_{r}\right)$ is contained in the intersection of $c l\left(\varpi_{r}+\sum_{i \in I} \mathbb{Z} \alpha_{i}\right)$ and the convex hull of $W \operatorname{cl}\left(\varpi_{r}\right)$.

(v) $W\left(\varpi_{r}\right)$ has a global crystal base.

(vi) Any irreducible finite-dimensional integrable $U_{q}^{\prime}(\mathfrak{g})$-module with cl $\left(\varpi_{r}\right)$ as an extremal weight is isomorphic to $W\left(\varpi_{r}\right)_{a}$ for some $a \in \mathbb{Q}\left(q_{s}\right)$.

We also need the following lemma that ensures the existence of the prepolarization on $W\left(\varpi_{r}\right)$.

Lemma 2.5 (33, 23] $) .\left(z_{r} u, z_{r} v\right)=(u, v)$ for $u, v \in V\left(\varpi_{r}\right)$.

Remark 2.1. This lemma is given as Proposition 7.3 of [33] and also as Lemma 4.7 of [23]. The lemmas or properties used to prove it hold for any affine algebra $\mathfrak{g}$.

Summing up the above discussions we have

Proposition 2.6. The fundamental representation $W\left(\varpi_{r}\right)$ has the following properties:

(i) $W\left(\varpi_{r}\right)$ has a polarization (, ).

(ii) There exists a $U_{q}^{\prime}(\mathfrak{g})_{\mathbb{Z}}$-submodule $W\left(\varpi_{r}\right)_{\mathbb{Z}}$ of $W\left(\varpi_{r}\right)$ such that

$$
\left(W\left(\varpi_{r}\right)_{\mathbb{Z}}, W\left(\varpi_{r}\right)_{\mathbb{Z}}\right) \subset \mathbb{Z}\left[q_{s}, q_{s}^{-1}\right] .
$$

Before finishing this section, let us mention the Drinfeld polynomials. It is known that irreducible finite-dimensional $U_{q}^{\prime}(\mathfrak{g})$-modules are classified by $\left|I_{0}\right|$-tuple of polynomials $\left\{P_{j}(u)\right\}_{j \in I_{0}}$ whose constant terms are 1. See e.g. [4. The degree of $P_{j}$ is given by $\left\langle\lambda, h_{j}\right\rangle$ where $\lambda$ is the highest weight of the corresponding module. Hence we have

Lemma 2.7. $W\left(\varpi_{r}\right)$ has the following Drinfeld polynomials

$$
P_{r}(u)=1-a_{r}^{\dagger} u, \quad P_{j}(u)=1 \text { for } j \neq r
$$

with some $a_{r}^{\dagger} \in \mathbb{Q}\left(q_{s}\right)$.

For types $A_{n}^{(1)}, D_{n}^{(1)}, E_{6,7,8}^{(1)}$ the explicit value of $a_{r}^{\dagger}$ is known [23, Remark 3.3].

\section{KR MODUles AND THE EXISTENCE OF CRYSTAL BASES}

3.1. Fusion construction. Let $V$ be a $U_{q}^{\prime}(\mathfrak{g})$-module. An $R$-matrix, denoted by $R(x, y)$, is an element of $\operatorname{Hom}_{U_{q}^{\prime}(\mathfrak{g})\left[x^{ \pm 1}, y^{ \pm 1}\right]}\left(V_{x} \otimes V_{y}, V_{y} \otimes V_{x}\right)$. For $V$ we assume the following:

(3.1) $V \otimes V$ is irreducible.

$$
\text { There exists } \lambda_{0} \in P_{c l} \text { such that wt } V \subset \lambda_{0}+\sum_{i \in I_{0}} \mathbb{Z}_{\leq 0} \alpha_{i} \text { and } \operatorname{dim} V_{\lambda_{0}}=1 \text {. }
$$

Under these assumptions it is known (see e.g. [13) that there exists a unique $R$ matrix up to multiple of a scalar function of $x, y$. Take a nonzero vector $u_{0}$ from $V_{\lambda_{0}}$. We normalize $R(x, y)$ in such a way that $R(x, y)\left(u_{0} \otimes u_{0}\right)=u_{0} \otimes u_{0}$. The normalized $R$-matrix is known to depend only on $x / y$. Because of the normalization, some matrix elements of $R(x, y)$ may have zeros or poles as a function of $x / y$. At the points $x / y=x_{0} / y_{0} \in \mathbb{Q}\left(q_{s}\right)$ where there is no zero or pole, $R\left(x_{0}, y_{0}\right)$ is an isomorphism.

Next we review the fusion construction following section 3 of 14 . Let $s$ be a positive integer and $\mathfrak{S}_{s}$ the $s$-th symmetric group. Let $s_{i}$ be the simple reflection 
which interchanges $i$ and $i+1$, and let $\ell(w)$ be the length of $w \in \mathfrak{S}_{s}$. Let $R(x, y)$ denote the $R$-matrix for $V_{x} \otimes V_{y}$. For any $w \in \mathfrak{S}_{s}$ we can construct a well-defined $\operatorname{map} R_{w}\left(x_{1}, \ldots, x_{s}\right): V_{x_{1}} \otimes \cdots \otimes V_{x_{s}} \rightarrow V_{x_{w(1)}} \otimes \cdots \otimes V_{x_{w(s)}}$ by

$$
\begin{aligned}
R_{1}\left(x_{1}, \ldots, x_{s}\right) & =1, \\
R_{s_{i}}\left(x_{1}, \ldots, x_{s}\right) & =\left(\bigotimes_{j<i} \operatorname{id}_{V_{x_{j}}}\right) \otimes R\left(x_{i}, x_{i+1}\right) \otimes\left(\bigotimes_{j>i+1} \operatorname{id}_{V_{x_{j}}}\right), \\
R_{w w^{\prime}}\left(x_{1}, \ldots, x_{s}\right) & =R_{w^{\prime}}\left(x_{w(1)}, \ldots, x_{w(s)}\right) \circ R_{w}\left(x_{1}, \ldots, x_{s}\right) \\
& \quad \text { for } w, w^{\prime} \text { such that } \ell\left(w w^{\prime}\right)=\ell(w)+\ell\left(w^{\prime}\right) .
\end{aligned}
$$

Fix $k \in d^{-1} \mathbb{Z} \backslash\{0\}$. Let us assume that

$$
\text { the normalized } R \text {-matrix } R(x, y) \text { does not have a pole at } x / y=q^{2 k} \text {. }
$$

For each $s \in \mathbb{Z}_{>0}$, we put

$$
\begin{aligned}
R_{s}= & R_{w_{0}}\left(q^{k(s-1)}, q^{k(s-3)}, \ldots, q^{-k(s-1)}\right): \\
& V_{q^{k(s-1)}} \otimes V_{q^{k(s-3)}} \otimes \cdots \otimes V_{q^{-k(s-1)}} \rightarrow V_{q^{-k(s-1)}} \otimes V_{q^{-k(s-3)}} \otimes \cdots \otimes V_{q^{k(s-1)}},
\end{aligned}
$$

where $w_{0}$ is the longest element of $\mathfrak{S}_{s}$. Then $R_{s}$ is a $U_{q}^{\prime}(\mathfrak{g})$-linear homomorphism. Define

$$
V_{s}=\operatorname{Im} R_{s}
$$

Let us denote by $W$ the image of

$$
R\left(q^{k}, q^{-k}\right): V_{q^{k}} \otimes V_{q^{-k}} \longrightarrow V_{q^{-k}} \otimes V_{q^{k}}
$$

and by $N$ its kernel. Then we have

$$
\begin{aligned}
& V_{s} \text { considered as a submodule of } V^{\otimes s}=V_{q^{-k(s-1)}} \otimes \cdots \otimes V_{q^{k(s-1)}} \\
& \text { is contained in } \bigcap_{i=0}^{s-2} V^{\otimes i} \otimes W \otimes V^{\otimes(s-2-i)} .
\end{aligned}
$$

Similarly, we have

$$
V_{s} \text { is a quotient of } V^{\otimes s} / \sum_{i=0}^{s-2} V^{\otimes i} \otimes N \otimes V^{\otimes(s-2-i)} .
$$

In the sequel, following [14] we define a prepolarization on $V_{s}$ and study necessary properties. First we recall the following lemma.

Lemma 3.1 ([14, Lemma 3.4.1]). Let $M_{j}$ and $N_{j}$ be $U_{q}^{\prime}(\mathfrak{g})$-modules and let $(,)_{j}$ be an admissible pairing between $M_{j}$ and $N_{j}(j=1,2)$. Then the pairing (, ) between $M_{1} \otimes M_{2}$ and $N_{1} \otimes N_{2}$ defined by $\left(u_{1} \otimes u_{2}, v_{1} \otimes v_{2}\right)=\left(u_{1}, v_{1}\right)_{1}\left(u_{2}, v_{2}\right)_{2}$ for all $u_{j} \in M_{j}$ and $v_{j} \in N_{j}$ is admissible.

Let $V$ be a finite-dimensional $U_{q}^{\prime}(\mathfrak{g})$-module satisfying (3.1) and (3.2). Suppose $V$ has a polarization. The polarization on $V$ gives an admissible pairing between $V_{x}$ and $V_{x^{-1}}$. Hence it induces an admissible pairing between $V_{x_{1}} \otimes \cdots \otimes V_{x_{s}}$ and $V_{x_{1}^{-1}} \otimes \cdots \otimes V_{x_{s}^{-1}}$

Lemma 3.2 ([14, Lemma 3.4.2]). If $x_{j}=x_{s+1-j}^{-1}$ for $j=1, \ldots, s$, then for any $u, u^{\prime} \in V_{x_{1}} \otimes \cdots \otimes V_{x_{s}}$, we have

$$
\left(u, R_{w_{0}}\left(x_{1}, \ldots, x_{s}\right) u^{\prime}\right)=\left(u^{\prime}, R_{w_{0}}\left(x_{1}, \ldots, x_{s}\right) u\right) .
$$


By taking $x_{i}=q^{k(s-2 i+1)}$, we obtain the admissible pairing $($,$) between W=$ $V_{q^{k(s-1)}} \otimes V_{q^{k(s-3)}} \otimes \cdots \otimes V_{q^{-k(s-1)}}$ and $W^{\prime}=V_{q^{-k(s-1)}} \otimes V_{q^{-k(s-3)}} \otimes \cdots \otimes V_{q^{k(s-1)}}$ that satisfies

$$
\left(w, R_{s} w^{\prime}\right)=\left(w^{\prime}, R_{s} w\right) \quad \text { for any } w, w^{\prime} \in W .
$$

This allows us to define a prepolarization $(,)_{s}$ on $V_{s}$ by

$$
\left(R_{s} u, R_{s} u^{\prime}\right)_{s}=\left(u, R_{s} u^{\prime}\right)
$$

for $u, u^{\prime} \in V_{q^{k(s-1)}} \otimes V_{q^{k(s-3)}} \otimes \cdots \otimes V_{q^{-k(s-1)}}$.

Assume

$$
V \text { admits a } U_{q}^{\prime}(\mathfrak{g})_{K_{\mathbb{Z}}} \text {-submodule } V_{K_{\mathbb{Z}}} \text { such that }\left(V_{K_{\mathbb{Z}}}\right)_{\lambda_{0}}=K_{\mathbb{Z}} u_{0} \text {. }
$$

Let us further set

$$
\left(V_{s}\right)_{K_{\mathbb{Z}}}=R_{s}\left(\left(V_{K_{\mathbb{Z}}}\right)^{\otimes s}\right) \cap\left(V_{K_{\mathbb{Z}}}\right)^{\otimes s} .
$$

Then [14, Proposition3.4.3] follows:

\section{Proposition 3.3.}

(i) $(,)_{s}$ is a nondegenerate prepolarization on $V_{s}$.

(ii) $\left(R_{s}\left(u_{0}^{\otimes s}\right), R_{s}\left(u_{0}^{\otimes s}\right)\right)_{s}=1$.

(iii) $\left(\left(V_{s}\right)_{K_{\mathbb{Z}}},\left(V_{s}\right)_{K_{\mathbb{Z}}}\right)_{s} \subset K_{\mathbb{Z}}$.

3.2. KR modules. We want to apply the fusion construction with $V$ being the fundamental representation $W\left(\varpi_{r}\right)$. Let us take $k$ to be $\left(\alpha_{r}, \alpha_{r}\right) / 2$ except in the case $k=1$ when $\mathfrak{g}=A_{2 n}^{(2)}$ and $r=n$.

Proposition 3.4. Assumptions (3.1), (3.2), (3.3), and (3.7) hold for the fundamental representations.

Proof. (3.1) is a consequence of Proposition 2.4(v) and the fact that $B\left(\varpi_{r}\right)$ is a "simple" crystal (see [17). (3.2) is valid by Proposition 2.4(iv) with $\lambda_{0}=c l\left(\varpi_{r}\right)$. Noting that $W\left(\varpi_{r}\right)$ is a "good" $U_{q}^{\prime}(\mathfrak{g})$-module, (3.3) is the consequence of Proposition 9.3 of [17. (3.7) is valid, since $W\left(\varpi_{r}\right)$ admits a $U_{q}^{\prime}(\mathfrak{g})_{\mathbb{Z}}$-submodule $W\left(\varpi_{r}\right)_{\mathbb{Z}}$ induced from $V\left(\varpi_{r}\right)_{\mathbb{Z}}$ such that $\left(W\left(\varpi_{r}\right)_{\mathbb{Z}}\right)_{c l\left(\varpi_{r}\right)}=\mathbb{Z}\left[q_{s}, q_{s}^{-1}\right] u_{\varpi_{r}}$.

For $r \in I_{0}$ and $s \in \mathbb{Z}_{>0}$ we define the $U_{q}^{\prime}(\mathfrak{g})$-module $W_{s}^{(r)}$ to be the module constructed by the fusion construction in section 3.1 with $V=W\left(\varpi_{r}\right)$ and $k=$ $\left(\alpha_{r}, \alpha_{r}\right) / 2$ except in the case $k=1$ when $\mathfrak{g}=A_{2 n}^{(2)}$ and $r=n$.

\section{Proposition 3.5.}

(i) There exists a prepolarization (, ) on $W_{s}^{(r)}$.

(ii) There exists a $U_{q}^{\prime}(\mathfrak{g})_{K_{\mathbb{Z}}}$-submodule $\left(W_{s}^{(r)}\right)_{K_{\mathbb{Z}}}$ of $W_{s}^{(r)}$ such that

$$
\left(\left(W_{s}^{(r)}\right)_{K_{\mathbb{Z}}},\left(W_{s}^{(r)}\right)_{K_{\mathbb{Z}}}\right) \subset K_{\mathbb{Z}} .
$$

(iii) There exists a vector $u_{0}$ of weight $s \varpi_{r}$ in $\left(W_{s}^{(r)}\right)_{K_{\mathbb{Z}}}$ such that $\left(u_{0}, u_{0}\right)=1$.

Proof. The results follow from Propositions 3.3 and 3.4 .

The following proposition is an easy consequence of the main result of Kashiwara [17. Note also that his result can be applied not only to KR modules but also to any irreducible modules. 
Proposition 3.6. $W_{s}^{(r)}$ is irreducible and its Drinfeld polynomials are given by

$$
P_{j}(u)= \begin{cases}\left(1-a_{r}^{\dagger} q_{r}^{1-s} u\right)\left(1-a_{r}^{\dagger} q_{r}^{3-s} u\right) \cdots\left(1-a_{r}^{\dagger} q_{r}^{s-1} u\right) & (j=r), \\ 1 & (j \neq r),\end{cases}
$$

except when $\mathfrak{g}=A_{2 n}^{(2)}$ and $r=n$. If $\mathfrak{g}=A_{2 n}^{(2)}$ and $r=n$, they are given by replacing $q_{r}$ with $q$ in the above formula.

Proof. Let $V$ be a nonzero submodule of $V_{s}=W_{s}^{(r)}$. To show the irreducibility, it suffices to show that any vector $v$ in $V_{s}$ is contained in $V$. By definition there exists a vector $u \in W\left(\varpi_{r}\right)^{\otimes s}$ such that $v=R_{s} u$. From Theorem 9.2(ii) of [17] we have $u_{0}^{\otimes s} \in V$. From Theorem 9.2(i) of loc. cit. there exists $x \in U_{q}^{\prime}(\mathfrak{g})$ such that $u=\Delta^{(s)}(x) u_{0}^{\otimes s}$, where $\Delta^{(s)}$ is the coproduct $U_{q}^{\prime}(\mathfrak{g}) \longrightarrow U_{q}^{\prime}(\mathfrak{g})^{\otimes s}$. Hence we have $v=R_{s} \Delta^{(s)}(x) u_{0}^{\otimes s}=\Delta^{(s)}(x) R_{s} u_{0}^{\otimes s}=\Delta^{(s)}(x) u_{0}^{\otimes s} \in V$.

Since $W_{s}^{(r)}$ is the irreducible module in $\left(W_{1}^{(r)}\right)_{q_{r}^{1-s}} \otimes\left(W_{1}^{(s)}\right)_{q_{r}^{3-s}} \otimes \cdots \otimes\left(W_{1}^{(r)}\right)_{q_{r}^{s-1}}$ generated by $u_{0}^{\otimes s}$, the latter statement is clear from [4, Corollary 3.5], Lemma 2.7 and the fact that if $V$ corresponds to $\left\{P_{j}(u)\right\}$, then $V_{a}$ corresponds to $\left\{P_{j}(a u)\right\}$.

This irreducible $U_{q}^{\prime}(\mathfrak{g})$-module $W_{s}^{(r)}$ is called Kirillov-Reshetikhin (KR) module:

Since the KR module $W_{s}^{(r)}$ is also a $U_{q}\left(\mathfrak{g}_{0}\right)$-module by restriction, we have the following direct sum decomposition as a $U_{q}\left(\mathfrak{g}_{0}\right)$-module:

$$
W_{s}^{(r)} \simeq \bigoplus_{\lambda \in \bar{P}_{+}} N_{s}^{(r)}(\lambda) \cdot \bar{V}(\lambda)
$$

Namely, $N_{s}^{(r)}(\lambda)$ is the multiplicity of the irreducible $U_{q}\left(\mathfrak{g}_{0}\right)$-module $\bar{V}(\lambda)$ in $W_{s}^{(r)}$. Then we have a criterion that the KR module has a crystal pseudobase.

Proposition 3.7. Suppose for any $\lambda \in \bar{P}_{+}$such that $N_{s}^{(r)}(\lambda)>0$ there exist $u(\lambda)_{j} \in\left(W_{s}^{(r)}\right)_{K_{\mathbb{Z}}}$ of weight $\lambda$ for $j=1, \ldots, N_{s}^{(r)}(\lambda)$. If we have $\left(u(\lambda)_{j}, u(\lambda)_{k}\right) \in$ $\delta_{j k}+q_{s} A$ and $\left(e_{j} u(\lambda)_{k}, e_{j} u(\lambda)_{k}\right) \in q_{s} q_{j}^{-2\left(1+\left\langle h_{j}, \lambda\right\rangle\right)} A$ for any $j \in I_{0}$, then $($,$) on$ $W_{s}^{(r)}$ is a polarization, and $W_{s}^{(r)}$ has a crystal pseudobase.

Proof. We use Proposition 2.1. All the assumptions except (2.4) are satisfied by Propositions 3.5. Note that $\left(u(\lambda)_{j}, u(\mu)_{k}\right)=0$ if $\lambda \neq \mu$.

Remark 3.1. From the previous proposition it immediately follows that if $W_{s}^{(r)}$ is irreducible as a $U_{q}\left(\mathfrak{g}_{0}\right)$-module, then it has a crystal pseudobase (see also [14, Proposition 3.4.4]). There is another case in which the existence of crystal pseudobase is proven for any $l$ and any $\mathfrak{g}$ except $A_{n}^{(1)}$ as in [14, Proposition 3.4.5]. It corresponds to $r=2$ when $\mathfrak{g}=B_{n}^{(1)}, D_{n}^{(1)}, A_{2 n-1}^{(1)}, r=6$ when $\mathfrak{g}=E_{6}^{(1)}$, and $r=1$ in all other cases. Here we follow the labeling of vertices of the Dynkin diagram by [12. We remark that the crystal base of $W_{1}^{(r)}$ for such $r$ is treated in [2].

There is an explicit formula of $N_{s}^{(r)}(\lambda)$ called the $(q=1)$ fermionic formula. We have [3, 7, 8, 9, 10, 20, 24, 25] for references. To explain it, we introduce $t_{i}$ and $t_{i}^{\vee}$ for $i \in I_{0}$ by

$$
t_{i}= \begin{cases}\frac{2}{\left(\alpha_{i}, \alpha_{i}\right)} & \text { if } \mathfrak{g} \text { is untwisted } \\ 1 & \text { if } \mathfrak{g} \text { is twisted }\end{cases}
$$


and $t_{i}^{\vee}=\left(t_{i}\right.$ for $\left.\mathfrak{g}^{\vee}\right)$, where $\mathfrak{g}^{\vee}$ is the dual Kac-Moody algebra to $\mathfrak{g}$. For $p \in \mathbb{Z}$ and $m \in \mathbb{Z}_{\geq 0}$ let $\left(\begin{array}{c}p+m \\ m\end{array}\right)$ stand for the binomial coefficient, i.e., $\left(\begin{array}{c}p+m \\ m\end{array}\right)=\prod_{k=1}^{m} \frac{p+k}{k}$. Then, for $r \in I_{0}, s \in \mathbb{Z}_{>0}$ and $\lambda \in \bar{P}_{+}$we have

$$
N_{s}^{(r)}(\lambda)=\sum_{\mathbf{m}} \prod_{a \in I_{0}, j \geq 1}\left(\begin{array}{c}
p_{j}^{(a)}+m_{j}^{(a)} \\
m_{j}^{(a)}
\end{array}\right),
$$

where

$$
p_{j}^{(a)}=\delta_{a i} \min (j, s)-\frac{1}{t_{a}^{\vee}} \sum_{b \in I_{0}, k \geq 1}\left(\alpha_{a}, \alpha_{b}\right) \min \left(t_{b} j, t_{a} k\right) m_{k}^{(b)}
$$

and the sum $\sum_{\mathbf{m}}$ is taken over all $\left(m_{j}^{(a)} \in \mathbb{Z}_{\geq 0} \mid a \in I_{0}, j \geq 1\right)$ satisfying

$$
\sum_{a \in I_{0}, j \geq 1} j m_{j}^{(a)} \alpha_{a}=s \varpi_{r}-\lambda .
$$

The proof of this formula goes as follows. Set $Q_{s}^{(r)}=\operatorname{ch} W_{s}^{(r)}$. It suffices to show that $Q_{s}^{(r)}=\sum_{\lambda \in \bar{P}_{+}} N_{s}^{(r)}(\lambda) \operatorname{ch} \bar{V}(\lambda)$. By Theorem 8.1 of [8] (see also Theorem 6.3 of [7] including the twisted cases), it suffices to show that $\left\{Q_{s}^{(r)}\right\}$ satisfies the conditions (A), (B), (C) in the theorem. (A) is evident by the construction of $W_{s}^{(r)}$, and (B), (C) were verified in 24, 9, 10, for the simply-laced, untwisted and twisted cases, respectively. Note that condition $(\mathrm{C})$ is replaced with another convergence property (4.15) of [21. Note also that there is an earlier result by Chari [3] for untwisted cases. It should also be noted that there is another explicit formula $M_{s}^{(r)}(\lambda)$ for the multiplicities $N_{s}^{(r)}(\lambda)$ which involves unsigned binomial coefficients, that is, $\left(\begin{array}{c}p+m \\ m\end{array}\right)=0$ if $p<0$ [8, 7]. It was recently shown by Di Francesco and Kedem [6] that $M_{s}^{(r)}(\lambda)=N_{s}^{(r)}(\lambda)$ in the untwisted cases.

For nonexceptional types, the explicit value of $N_{s}^{(r)}(\lambda)$ can be found in Section 7 of [8] for untwisted cases, and in section 6.2 of [7] for twisted cases. See (4.1).

\section{EXISTENCE OF CRYSTAL PSEUdObASES FOR NONEXCEPTIONAL TYPES}

In this section we show that any KR module for nonexceptional type has a crystal pseudobase. For type $A_{n}^{(1)}$ this fact is established in [14. So we do not deal with the $A_{n}^{(1)}$ case.

4.1. Dynkin data. First we list the Dynkin diagrams of all nonexceptional affine algebras except $A_{n}^{(1)}$ in Table 1. We also list the pair $\left(\nu, \mathfrak{g}_{0}\right)$ in the table with a partition $\nu=\Theta, \square, \square$ and a simple Lie algebra $\mathfrak{g}_{0}$ whose Dynkin diagram is the one obtained by removing the 0 -vertex. Note that the difference of $\nu$ comes from the diagram near the 0 -vertex.

The simple roots for type $B_{n}, C_{n}, D_{n}$ are

$$
\begin{aligned}
& \alpha_{i}=\epsilon_{i}-\epsilon_{i+1} \quad \text { for } 1 \leq i<n, \\
& \alpha_{n}= \begin{cases}\epsilon_{n-1}+\epsilon_{n} & \text { for type } D_{n}, \\
\epsilon_{n} & \text { for type } B_{n}, \\
2 \epsilon_{n} & \text { for type } C_{n},\end{cases}
\end{aligned}
$$




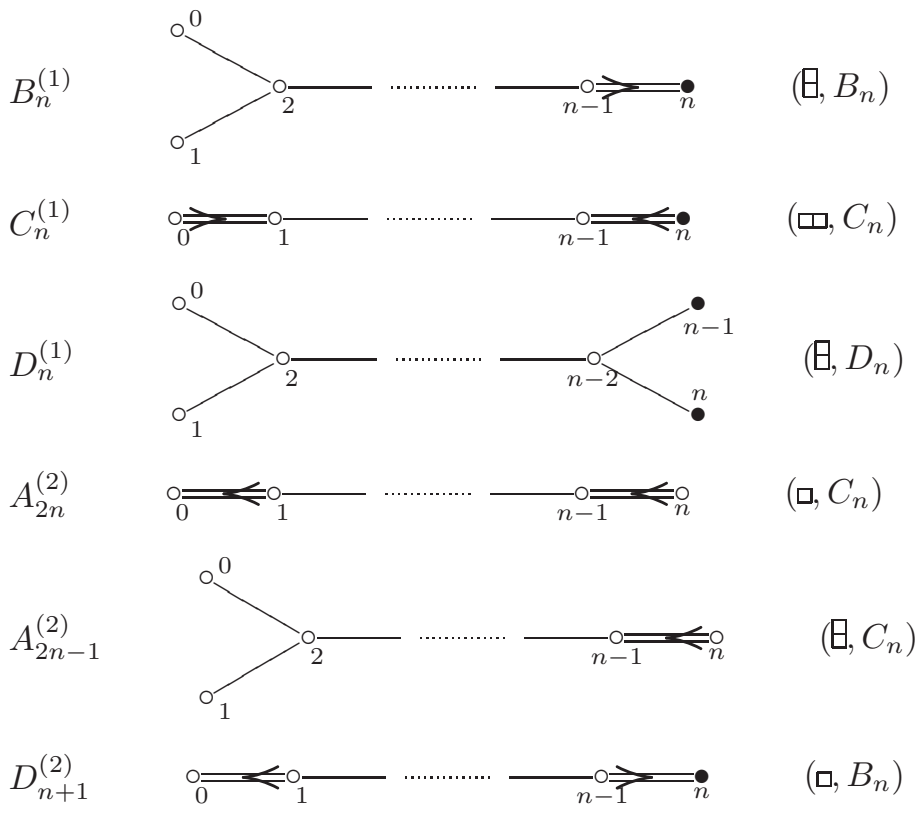

TABLE 1. Dynkin diagrams

and the fundamental weights are

$$
\begin{array}{lll}
\text { Type } D_{n}: & \varpi_{i}=\epsilon_{1}+\cdots+\epsilon_{i} & \text { for } 1 \leq i \leq n-2, \\
& \varpi_{n-1}=\left(\epsilon_{1}+\cdots+\epsilon_{n-1}-\epsilon_{n}\right) / 2, & \\
& \varpi_{n}=\left(\epsilon_{1}+\cdots+\epsilon_{n-1}+\epsilon_{n}\right) / 2 ; & \\
\text { Type } B_{n}: & \varpi_{i}=\epsilon_{1}+\cdots+\epsilon_{i} & \text { for } 1 \leq i \leq n-1, \\
& \varpi_{n}=\left(\epsilon_{1}+\cdots+\epsilon_{n-1}+\epsilon_{n}\right) / 2 ; & \\
\text { Type } C_{n}: & \varpi_{i}=\epsilon_{1}+\cdots+\epsilon_{i} & \text { for } 1 \leq i \leq n,
\end{array}
$$

where $\epsilon_{i}(i=1, \ldots, n)$ are vectors in the weight space of each simple Lie algebra. (By convention we set $\varpi_{0}=0$.) These elements can be viewed as those of the weight lattice $P$ of the affine algebra in Table 1. On $P$ we defined the inner product $($, normalized as $(\delta, \lambda)=\langle c, \lambda\rangle$ for $\lambda \in P$. This normalization is equivalent to setting $\left(\epsilon_{i}, \epsilon_{j}\right)=\kappa \delta_{i j}$ with $\kappa=\frac{1}{2}$ for $C_{n}^{(1)},=2$ for $D_{n+1}^{(2)}$, and $=1$ for the other types. However, in this section we renormalize it by $\left(\epsilon_{i}, \epsilon_{j}\right)=\delta_{i j}$. This is equivalent to setting $\left(\alpha_{i}, \alpha_{i}\right) / 2=1$ for $i$ not an end node of the Dynkin diagram. We also note that

$$
\alpha_{0}= \begin{cases}\delta-\epsilon_{1}-\epsilon_{2} & \text { if } \nu=\theta, \\ \delta-2 \epsilon_{1} & \text { if } \nu=\text { 口, } \\ \delta-\epsilon_{1} & \text { if } \nu=\square .\end{cases}
$$

4.2. Existence of crystal pseudobases for $\mathbf{K R}$ modules. We first present the branching rule of KR modules of affine type listed in Table 1 with respect to the subalgebra $U_{q}\left(\mathfrak{g}_{0}\right)$. They can be found in [8, Theorems 7.1 and 8.1] and 
[7. Theorems 6.2 and 6.3]. For $i \in I_{0}$ for $\mathfrak{g}$ we say $i$ is a spin node if the vertex $i$ is filled in Table 1. If $r \in I_{0}$ is a spin node, then the $\mathrm{KR}$ module $W_{s}^{(r)}$ is irreducible as a $U_{q}\left(\mathfrak{g}_{0}\right)$-module:

$$
W_{s}^{(r)} \simeq \bar{V}\left(s \varpi_{r}\right) .
$$

Suppose now that $r \in I_{0}$ is not a spin node. Let $\omega$ be a dominant integral weight of the form of $\omega=\sum_{i} c_{i} \varpi_{i}$. Assume $c_{i}=0$ for $i$ a spin node. In the standard way we represent $\omega$ by the partition that has exactly $c_{i}$ columns of height $i$. Then the KR module $W_{s}^{(r)}$ decomposes into

$$
W_{s}^{(r)} \simeq \bigoplus_{\omega} \bar{V}(\omega)
$$

as a $U_{q}\left(\mathfrak{g}_{0}\right)$-module, where $\omega$ runs over all partitions that can be obtained from the $r \times s$ rectangle by removing pieces of shape $\nu$ (with $\nu$ as in Table 1).

If $r \in I_{0}$ is a spin node, the KR module $W_{s}^{(r)}$ has a crystal pseudobase by Remark 3.1. Suppose $r$ is not a spin node. As we have seen, we have $N_{s}^{(r)}(\lambda) \leq 1$. Hence, by Proposition 3.7, in order to show the existence of crystal pseudobase, it suffices to define a vector $u(\lambda) \in\left(W_{s}^{(r)}\right)_{K_{\mathbb{Z}}}$ of weight $\lambda$ for any $\lambda$ such that $N_{s}^{(r)}=1$, and show $(u(\lambda), u(\lambda)) \in 1+q_{s} A$ and $\left(e_{j} u(\lambda), e_{j} u(\lambda)\right) \in q_{s} q_{j}^{-2\left(1+\left\langle h_{j}, \lambda\right\rangle\right)} A$ for $j \in I_{0}$. In the subsequent subsections, we do this task by dividing it into 3 cases according to the shape of $\nu$.

4.3. Calculation of prepolarization: $D_{n}^{(1)}, B_{n}^{(1)}, A_{2 n-1}^{(2)}$ cases. We assume $1 \leq$ $r \leq n-2$ for $D_{n}^{(1)}, 1 \leq r \leq n-1$ for $B_{n}^{(1)}$ and $1 \leq r \leq n$ for $A_{2 n-1}^{(2)}$. Let $r^{\prime}=[r / 2]$. Let $\mathbf{c}=\left(c_{1}, c_{2}, \ldots, c_{r^{\prime}}\right)$ be a sequence of integers such that $s \geq c_{1} \geq c_{2} \geq \cdots \geq$ $c_{r^{\prime}} \geq 0$. For such $\mathbf{c}$ we define a vector $u_{m}\left(0 \leq m \leq r^{\prime}\right)$ in $W_{s}^{(r)}$ inductively by

$$
u_{m}=\left(e_{r-2 m}^{\left(c_{m}\right)} \cdots e_{2}^{\left(c_{m}\right)} e_{1}^{\left(c_{m}\right)}\right)\left(e_{r-2 m+1}^{\left(c_{m}\right)} \cdots e_{3}^{\left(c_{m}\right)} e_{2}^{\left(c_{m}\right)}\right) e_{0}^{\left(c_{m}\right)} u_{m-1},
$$

where $u_{0}$ is the vector in (iii) of Proposition 3.5 Set $u(\mathbf{c})=u_{r^{\prime}}$. The weight of $u(\mathbf{c})$ is given by

$$
\lambda(\mathbf{c})=\sum_{j=0}^{r^{\prime}}\left(c_{j}-c_{j+1}\right) \varpi_{r-2 j},
$$

where we have set $c_{0}=s, c_{r^{\prime}+1}=0$, and $\varpi_{0}$ should be understood as $0 . \lambda(\mathbf{c})$ represents all $\omega$ in (4.1) when c runs over all possible sequences. For $l, m \in \mathbb{Z}_{\geq 0}$ such that $m \leq l$ we define the $q$-binomial coefficient by

$$
\left[\begin{array}{c}
l \\
m
\end{array}\right]=\frac{[l] !}{[m] ![l-m] !} .
$$

The following proposition calculates values of the prepolarization $($,$) on W_{s}^{(r)}$.

\section{Proposition 4.1.}

(1) $(u(\mathbf{c}), u(\mathbf{c}))=\prod_{j=1}^{r^{\prime}} q^{c_{j}\left(2 s-c_{j}\right)}\left[\begin{array}{l}2 s \\ c_{j}\end{array}\right]$,

(2) $\left(e_{j} u(\mathbf{c}), e_{j} u(\mathbf{c})\right)=0$ unless $r-j \in 2 \mathbb{Z}_{\geq 0}$. If $r-j \in 2 \mathbb{Z}_{\geq 0}$, then setting $p=(r-j) / 2+1,\left(e_{j} u(\mathbf{c}), e_{j} u(\mathbf{c})\right)$ is given by

$$
q^{2 s-c_{p-1}-1}\left[2 s-c_{p-1}\right] \prod_{j=1}^{r^{\prime}} q^{\left(c_{j}-\delta_{j, p}\right)\left(2 s-c_{j}\right)}\left[\begin{array}{l}
2 s-\delta_{j, p} \\
c_{j}-\delta_{j, p}
\end{array}\right] .
$$


For type $D_{n}^{(1)}$ this proposition is proven in [27]. The proof goes completely parallel also for type $B_{n}^{(1)}$ and $A_{2 n-1}^{(2)}$. Note that $q_{i}=q$ for $i \neq n, q_{n}=q, q^{1 / 2}, q^{2}$ for $D_{n}^{(1)}, B_{n}^{(1)}, A_{2 n-1}^{(2)}$, respectively, and $q_{s}=q^{1 / 2}$ for $B_{n}^{(1)},=q$ for $D_{n}^{(1)}, A_{2 n-1}^{(2)}$. Since $q^{m-1}[m], q^{n(m-n)} \in 1+q A$ and $\left\langle h_{j}, \lambda(\mathbf{c})\right\rangle=c_{p-1}-c_{p} \geq 0$, we have $(u(\mathbf{c}), u(\mathbf{c})) \in$ $1+q_{s} A$ and $\left(e_{j} u(\mathbf{c}), e_{j} u(\mathbf{c})\right) \in q_{s} q_{j}^{-2\left(1+\left\langle h_{j}, \lambda(\mathbf{c})\right\rangle\right)} A$, for $j \in I_{0}$. This establishes the conditions of Proposition 3.7 and hence proves Theorem 1.1 that $W_{s}^{(r)}$ has a crystal pseudobase.

We denote the crystal of $W_{s}^{(r)}$ by $B^{r, s}$. Similar to $\mathfrak{g}_{0}$ one can consider $\mathfrak{g}_{1}$, which is another (mutually isomorphic) simple Lie algebra obtained by removing the vertex 1 from the Dynkin diagram of $\mathfrak{g}$. The following proposition will be used to show that $B^{r, s}$ is isomorphic to $\tilde{B}^{r, s}$, which is given combinatorially in the next section.

Proposition 4.2. Let $1 \leq r \leq n-2$ for $\mathfrak{g}=D_{n}^{(1)}, 1 \leq r \leq n-1$ for $\mathfrak{g}=B_{n}^{(1)}$, $1 \leq r \leq n$ for $\mathfrak{g}=A_{2 n-1}^{(2)}$, and $s \in \mathbb{Z}_{>0}$. Then for $i=0,1, B^{r, s}$ decomposes as $U_{q}\left(\mathfrak{g}_{i}\right)$-crystals into

$$
B^{r, s} \simeq \bigoplus_{0 \leq m_{1} \leq \cdots \leq m_{s} \leq[r / 2]} B^{\mathfrak{g}_{i}}\left(\sigma^{i}\left(\varpi_{r-2 m_{1}}+\cdots+\varpi_{r-2 m_{s}}\right)\right) .
$$

Here $B^{\mathfrak{g}_{i}}(\lambda)$ is the crystal base of the highest weight $U_{q}\left(\mathfrak{g}_{i}\right)$-module of highest weight $\lambda$, and $\sigma$ is the automorphism on $P$ such that $\sigma\left(\Lambda_{0}\right)=\Lambda_{1}, \sigma\left(\Lambda_{1}\right)=\Lambda_{0}, \sigma\left(\Lambda_{j}\right)=\Lambda_{j}$ $(j>1)$ and extended linearly.

Proof. If $i=0$, the claim is a direct consequence of (4.1). For $i=1$ note that the Weyl group of $\mathfrak{g}_{0}$ contains an element $w$ which sends $\varpi_{j}$ to $\sigma\left(\varpi_{j}\right)$ for any $j$ such that $0 \leq j \leq r$, where by convention $\varpi_{0}=0$. (Using the orthogonal basis $\left\{\epsilon_{i}\right\}$ of section 4.1 of the weight space of $\mathfrak{g}_{0}$, we can take an element $w$ such that $w\left(\epsilon_{i}\right)=(-1)^{\delta(i)} \epsilon_{i}$, where $\delta(i)=1$ if $i=1, n$ for $\mathfrak{g}=D_{n}^{(1)}, i=1$ for $\mathfrak{g}=B_{n}^{(1)}$ and $A_{2 n-1}^{(2)}$, and $\delta(i)=0$ otherwise.) Since $W_{s}^{(r)}$ is a direct sum also as a $U_{q}\left(\mathfrak{g}_{1}\right)$-module, it is enough to show the following equality of characters.

$$
\operatorname{ch} W_{s}^{(r)}=\sum_{0 \leq m_{1} \leq \cdots \leq m_{s} \leq[r / 2]} \operatorname{ch} V^{\mathfrak{g}_{1}}\left(\sigma\left(\varpi_{r-2 m_{1}}+\cdots+\varpi_{r-2 m_{s}}\right)\right)
$$

Here $V^{\mathfrak{g}_{1}}(\lambda)$ denotes the highest weight $U_{q}\left(\mathfrak{g}_{1}\right)$-module of highest weight $\lambda$. But noting $w\left(\alpha_{0}\right)=\alpha_{1}, w\left(\alpha_{1}\right)=\alpha_{0}, w\left(\alpha_{j}\right)=\alpha_{j}(j>1)$ on $P_{c l}$, 4.3) is shown from

$$
\operatorname{ch} W_{s}^{(r)}=\sum_{0 \leq m_{1} \leq \cdots \leq m_{s} \leq[r / 2]} \operatorname{ch} V^{\mathfrak{g}_{0}}\left(\varpi_{r-2 m_{1}}+\cdots+\varpi_{r-2 m_{s}}\right)
$$

since $w$ preserves the weight multiplicity.

4.4. Calculation of prepolarization: $C_{n}^{(1)}$ case. We assume $1 \leq r \leq n-1$. Let $\mathbf{c}=\left(c_{1}, c_{2}, \ldots, c_{r}\right)$ be a sequence of integers such that $[s / 2] \geq c_{1} \geq c_{2} \geq \cdots \geq c_{r} \geq$ 0 . For such $\mathbf{c}$ we define a vector $u_{m}(0 \leq m \leq r)$ in $W_{s}^{(r)}$ inductively by

$$
u_{m}=e_{r-m}^{\left(2 c_{m}\right)} \cdots e_{2}^{\left(2 c_{m}\right)} e_{1}^{\left(2 c_{m}\right)} e_{0}^{\left(c_{m}\right)} u_{m-1},
$$

where $u_{0}$ is the vector in (iii) of Proposition 3.5. Set $u(\mathbf{c})=u_{r}$. The weight of $u(\mathbf{c})$ is given by

$$
\lambda(\mathbf{c})=\sum_{j=0}^{r} 2\left(c_{j}-c_{j+1}\right) \varpi_{r-j},
$$


where we have set $c_{0}=s / 2, c_{r+1}=0$, and $\varpi_{0}$ should be understood as $0 . \quad \lambda(\mathbf{c})$ represents all $\omega$ in (4.1) when $\mathbf{c}$ runs over all possible sequences. In this subsection, besides (4.2) we also use $\left[\begin{array}{c}l \\ m\end{array}\right]_{0}$ defined by (4.2) with $q$ replaced by $q_{0}=q^{2}$. (Recall that we have renormalized the inner product $($,$) on P$ in such a way that $\left(\epsilon_{i}, \epsilon_{j}\right)=$ $\delta_{i j}$.)

We are to calculate the values of $(u(\mathbf{c}), u(\mathbf{c}))$ and $\left(e_{j} u(\mathbf{c}), e_{j} u(\mathbf{c})\right)$. Since the calculation goes parallel to the case of $D_{n}^{(1)}$ treated in [27, we only give here intermediate results as a lemma. We write $\|u\|^{2}$ for $(u, u)$.

\section{Lemma 4.3.}

(1) $\left\|u_{m}\right\|^{2}=q_{0}^{c_{m}\left(s-c_{m}\right)}\left[\begin{array}{c}s \\ c_{m}\end{array}\right]_{0}\left\|u_{m-1}\right\|^{2}$

(2) $e_{j} u(\mathbf{c})=0$ if $j>r$

(3) $\left\|e_{j} u(\mathbf{c})\right\|^{2}=q^{2 \beta_{j}}\left\|f_{j} u(\mathbf{c})\right\|^{2}+q^{\beta_{j}-1}\left[\beta_{j}\right]\|u(\mathbf{c})\|^{2}$ if $1 \leq j \leq r$, where $\beta_{j}=$ $-\left\langle h_{j}, \lambda(\mathbf{c})\right\rangle=2\left(c_{r+1-j}-c_{r-j}\right)$,

(4)

$$
\begin{aligned}
\left\|f_{j} u(\mathbf{c})\right\|^{2}= & \prod_{\substack{1 \leq m \leq r \\
m \neq r-j+1}} q_{0}^{c_{m}\left(s-c_{m}\right)}\left[\begin{array}{c}
s \\
c_{m}
\end{array}\right]_{0} \\
& \times q_{0}^{c_{r-j+1}\left(s-1-c_{r-j+1}\right)}\left[\begin{array}{c}
s-1 \\
c_{r-j+1}
\end{array}\right]_{0} \times q^{2 c_{r-j}-1}\left[2 c_{r-j}\right] .
\end{aligned}
$$

From this lemma we have

\section{Proposition 4.4 .}

(1) $(u(\mathbf{c}), u(\mathbf{c}))=\prod_{m=1}^{r} q^{c_{m}\left(s-c_{m}\right)}\left[\begin{array}{c}s \\ c_{m}\end{array}\right]_{0}$,

$$
\left(e_{j} u(\mathbf{c}), e_{j} u(\mathbf{c})\right)= \begin{cases}q^{2 s-2 c_{r-j}-1}\left[2 s-2 c_{r-j}\right] \\
\times \prod_{m=1}^{r} q_{0}^{\left(c_{m}-\delta_{m, r-j+1}\right)\left(s-c_{m}\right)}\left[\begin{array}{cc}
s-\delta_{m, r-j+1} \\
c_{m}-\delta_{m, r-j+1}
\end{array}\right] & \text { if } 1 \leq j \leq r, \\
0 & \text { if } r<j \leq n .\end{cases}
$$

Note that $q_{i}=q$ for $i \neq 0, n, q_{n}=q^{2}$, and $q_{s}=q$ under the renormalization. Since $\left\langle h_{j}, \lambda(\mathbf{c})\right\rangle=-\beta_{j}=2\left(c_{r-j}-c_{r+1-j}\right) \geq 0$, we have $(u(\mathbf{c}), u(\mathbf{c})) \in 1+q_{s} A$ and $\left(e_{j} u(\mathbf{c}), e_{j} u(\mathbf{c})\right) \in q_{s} q_{j}^{-2\left(1+\left\langle h_{j}, \lambda(\mathbf{c})\right\rangle\right)} A$ for $j \in I_{0}$. By Proposition 3.7 this proves Theorem 1.1

4.5. Calculation of prepolarization: $A_{2 n}^{(2)}, D_{n+1}^{(2)}$ cases. We assume $1 \leq r \leq n$ for $A_{2 n}^{(2)}$ and $1 \leq r \leq n-1$ for $D_{n+1}^{(2)}$. Let $\mathbf{c}=\left(c_{1}, c_{2}, \ldots, c_{r}\right)$ be a sequence of integers such that $s \geq c_{1} \geq c_{2} \geq \cdots \geq c_{r} \geq 0$. For such $\mathbf{c}$ we define a vector $u_{m}$ $(0 \leq m \leq r)$ in $W_{s}^{(r)}$ inductively by

$$
u_{m}=e_{r-m}^{\left(c_{m}\right)} \cdots e_{1}^{\left(c_{m}\right)} e_{0}^{\left(c_{m}\right)} u_{m-1},
$$

where $u_{0}$ is the vector in (iii) of Proposition 3.5. Set $u(\mathbf{c})=u_{r}$. The weight of $u(\mathbf{c})$ is given by

$$
\lambda(\mathbf{c})=\sum_{j=0}^{r}\left(c_{j}-c_{j+1}\right) \varpi_{r-j}
$$


where we have set $c_{0}=s, c_{r+1}=0$, and $\varpi_{0}$ should be understood as $0 . \lambda(\mathbf{c})$ represents all $\omega$ in (4.1) when c runs over all possible sequences. In this subsection, besides (4.2) we also use $\left[\begin{array}{l}l \\ m\end{array}\right]_{0}$ defined by (4.2) with $q$ replaced by $q_{0}=q^{1 / 2}$.

As in the previous subsection, we only give here intermediate results as a lemma. As before we write $\|u\|^{2}$ for $(u, u)$.

\section{Lemma 4.5.}

(1) $\left\|u_{m}\right\|^{2}=q_{0}^{c_{m}\left(2 s-c_{m}\right)}\left[\begin{array}{c}2 s \\ c_{m}\end{array}\right]_{0}\left\|u_{m-1}\right\|^{2}$,

(2) $e_{j} u(\mathbf{c})=0$ if $j>r$

(3) $\left\|e_{j} u(\mathbf{c})\right\|^{2}=q^{2 \beta_{j}}\left\|f_{j} u(\mathbf{c})\right\|^{2}+q^{\beta_{j}-1}\left[\beta_{j}\right]\|u(\mathbf{c})\|^{2}$ if $1 \leq j \leq r$, where $\beta_{j}=$ $-\left\langle h_{j}, \lambda(\mathbf{c})\right\rangle=c_{r+1-j}-c_{r-j}$,

$$
\begin{aligned}
\left\|f_{j} u(\mathbf{c})\right\|^{2}= & \prod_{m=1}^{r} q_{0}^{c_{m}\left(2 s-2 \delta^{(1)}-c_{m}\right)}\left[\begin{array}{c}
2 s-2 \delta^{(1)} \\
c_{m}
\end{array}\right]_{0} \times q^{c_{r-j}-1}\left[c_{r-j}\right] \\
+ & \prod_{m=1}^{r} q_{0}^{\left(c_{m}+\delta^{(1)}-\delta^{(2)}\right)\left(2 s-\delta^{(1)}+\delta^{(2)}-c_{m}\right)}\left[\begin{array}{c}
2 s-2 \delta^{(1)} \\
c_{m}-\delta^{(1)}-\delta^{(2)}
\end{array}\right]_{0} \\
& \times\left[2 s-c_{r-j}+1\right]_{0}^{2},
\end{aligned}
$$

where $\delta^{(1)}=\delta_{m, r-j+1}, \delta^{(2)}=\delta_{m, r-j}$.

From this lemma we have

\section{Proposition 4.6.}

(1) $(u(\mathbf{c}), u(\mathbf{c}))=\prod_{m=1}^{r} q^{c_{m}\left(2 s-c_{m}\right)}\left[\begin{array}{c}2 s \\ c_{m}\end{array}\right]_{0}$,

$$
\left(e_{j} u(\mathbf{c}), e_{j} u(\mathbf{c})\right)= \begin{cases}q^{2 \beta_{j}}\left\|f_{j} u(\mathbf{c})\right\|^{2}+q^{\beta_{j}-1}\left[\beta_{j}\right]\|u(\mathbf{c})\|^{2} & \text { if } 1 \leq j \leq r, \\ 0 & \text { if } r<j \leq n,\end{cases}
$$

where $\beta_{j}$ and $\left\|f_{j} u(\mathbf{c})\right\|^{2}$ are given in the previous lemma.

Note that $q_{i}=q$ for $i \neq 0, n, q_{n}=q^{2}$ for $A_{2 n}^{(2)},=q^{1 / 2}$ for $D_{n+1}^{(2)}$, and $q_{s}=q^{1 / 2}$ under the renormalization. Since $\left\langle h_{j}, \lambda(\mathbf{c})\right\rangle=-\beta_{j}=c_{r-j}-c_{r+1-j} \geq 0$, we have $(u(\mathbf{c}), u(\mathbf{c})) \in 1+q_{s} A$ and $\left(e_{j} u(\mathbf{c}), e_{j} u(\mathbf{c})\right) \in q_{s} q_{j}^{-2\left(1+\left\langle h_{j}, \lambda(\mathbf{c})\right\rangle\right)} A$ for $j \in I_{0}$. By Proposition 3.7 this proves Theorem 1.1 .

\section{Combinatorial CRystal $\tilde{B}^{r, s}$ of type $D_{n}^{(1)}, B_{n}^{(1)}, A_{2 n-1}^{(2)}$}

In this section we review the combinatorial crystal $\tilde{B}^{r, s}$ of $\left[30,32\right.$ of type $D_{n}^{(1)}$, $B_{n}^{(1)}$, and $A_{2 n-1}^{(2)}$ and prove some preliminary results that will be needed in Section 6 to establish the equivalence of $\tilde{B}^{r, s}$ and $B^{r, s}$.

5.1. Type $D_{n}, B_{n}$, and $C_{n}$ crystals. Crystals associated with a $U_{q}(\mathfrak{g})$-module when $\mathfrak{g}$ is a simple Lie algebra of nonexceptional type, were studied by Kashiwara and Nakashima [18. Here we review the combinatorial structure in terms of tableaux of the crystals of type $X_{n}=D_{n}, B_{n}$, and $C_{n}$ since these are the finite subalgebras relevant to the KR crystals of type $D_{n}^{(1)}, B_{n}^{(1)}$, and $A_{2 n-1}^{(2)}$.

For $\mathfrak{g}=D_{n}^{(1)}, B_{n}^{(1)}$, or $A_{2 n-1}^{(2)}$, any $\mathfrak{g}_{0}$ dominant weight $\omega$ without a spin component can be expressed as $\omega=\sum_{i} c_{i} \varpi_{i}$ for nonnegative integers $c_{i}$ and the sum 


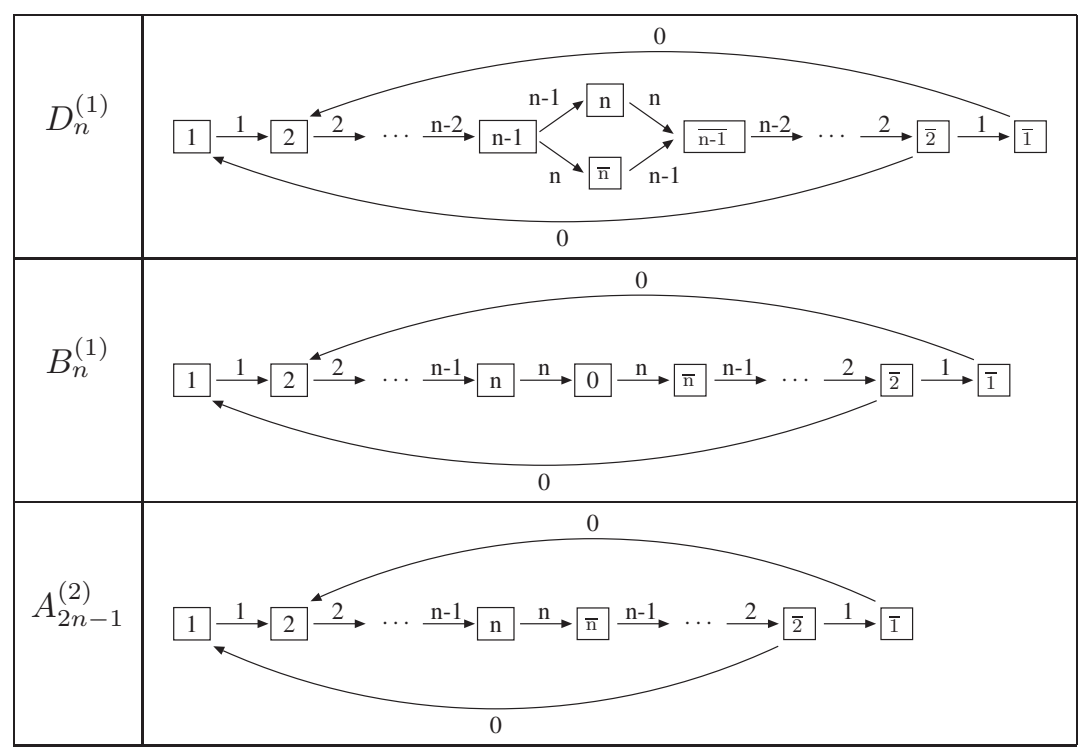

TABLE 2. KR crystal $B^{1,1}$

runs over all $i=1,2, \ldots, n$ not a spin node. As explained earlier we represent $\omega$ by the partition that has exactly $c_{i}$ columns of height $i$. For type $D_{n}$, this can be extended by associating a column of height $n-1$ with $\varpi_{n-1}+\varpi_{n}$ and a column of height $n$ with $2 \varpi_{n}$. For type $B_{n}$ one may associate a column of height $n$ with $2 \varpi_{n}$. Conversely, if $\omega$ is a partition, we write $c_{i}(\omega)$ for the number of columns of $\omega$ of height $i$. From now on we identify partitions and dominant weights in this way.

The crystal graph $B\left(\varpi_{1}\right)$ of the vector representation for type $D_{n}, B_{n}$, and $C_{n}$ is given in Table 2 by removing the 0 arrows in the crystal $B^{1,1}$ of type $D_{n}^{(1)}, B_{n}^{(1)}$, and $A_{2 n-1}^{(2)}$, respectively. The crystal $B\left(\varpi_{\ell}\right)$ for $\ell$ not a spin node can be realized as the connected component of $B\left(\varpi_{1}\right)^{\otimes \ell}$ containing the element $\ell \otimes(\ell-1) \otimes \cdots \otimes 1$, where we use the anti-Kashiwara convention for tensor products. Similarly, the crystal $B(\omega)$ labeled by a dominant weight $\omega=\varpi_{\ell_{1}}+\cdots+\varpi_{\ell_{k}}$ with $\ell_{1} \geq \ell_{2} \geq$ $\cdots \geq \ell_{k}$ not containing spin nodes can be realized as the connected component in $B\left(\varpi_{\ell_{1}}\right) \otimes \cdots \otimes B\left(\varpi_{\ell_{k}}\right)$ containing the element $u_{\varpi_{\ell_{1}}} \otimes \cdots \otimes u_{\varpi_{\ell_{k}}}$, where $u_{\varpi_{i}}$ is the highest weight element in $B\left(\varpi_{i}\right)$. As shown in [18, the elements of $B(\omega)$ can be labeled by tableaux of shape $\omega$ in the alphabet $\{1,2, \ldots, n, \bar{n}, \ldots, \overline{1}\}$ for types $D_{n}$ and $C_{n}$ and the alphabet $\{1,2, \ldots, n, 0, \bar{n}, \ldots, \overline{1}\}$ for type $B_{n}$. For the explicit rules of type $D_{n}, B_{n}$, and $C_{n}$ tableaux we refer the reader to [18; see also [11.

5.2. Definition of $\tilde{B}^{r, s}$. Let $\mathfrak{g}$ be of type $D_{n}^{(1)}, B_{n}^{(1)}$, or $A_{2 n-1}^{(2)}$ with the underlying finite Lie algebra $\mathfrak{g}_{0}$ of type $X_{n}=D_{n}, B_{n}$, or $C_{n}$, respectively. The combinatorial crystal $\tilde{B}^{r, s}$ is defined as follows. As an $X_{n}$-crystal, $\tilde{B}^{r, s}$ decomposes into the following irreducible components

$$
\tilde{B}^{r, s} \cong \bigoplus_{\omega} B(\omega)
$$

for $1 \leq r \leq n$ not a spin node. Here $B(\omega)$ is the $X_{n}$-crystal of highest weight $\omega$ and the sum runs over all dominant weights $\omega$ that can be obtained from $s \varpi_{r}$ by 
the removal of vertical dominoes, where $\varpi_{i}$ are the fundamental weights of $X_{n}$ as defined in section 5.1. The additional operators $\tilde{e}_{0}$ and $\tilde{f}_{0}$ are defined as

$$
\begin{aligned}
& \tilde{f}_{0}=\sigma \circ \tilde{f}_{1} \circ \sigma, \\
& \tilde{e}_{0}=\sigma \circ \tilde{e}_{1} \circ \sigma,
\end{aligned}
$$

where $\sigma$ is the crystal analogue of the automorphism of the Dynkin diagram that interchanges nodes 0 and 1. The involution $\sigma$ is defined in Definition 5.1

5.3. Definition of $\sigma$. To define $\sigma$ we first need the notion of \pm diagrams. A \pm diagram $P$ of shape $\Lambda / \lambda$ is a sequence of partitions $\lambda \subset \mu \subset \Lambda$ such that $\Lambda / \mu$ and $\mu / \lambda$ are horizontal strips. We depict this \pm diagram by the skew tableau of shape $\Lambda / \lambda$ in which the cells of $\mu / \lambda$ are filled with the symbol + and those of $\Lambda / \mu$ are filled with the symbol - . Write $\Lambda=\operatorname{outer}(P)$ and $\lambda=\operatorname{inner}(P)$ for the outer and inner shapes of the \pm diagram $P$. For type $A_{2 n-1}^{(2)}$ and $r=n$, the inner shape $\lambda$ is not allowed to be of height $n$. When drawing partitions or tableaux, we use the French convention where the parts are drawn in increasing order from top to bottom.

There is a bijection $\Phi: P \mapsto b$ from \pm diagrams $P$ of shape $\Lambda / \lambda$ to the set of $X_{n-1}$-highest weight vectors $b$ of $X_{n-1}$-weight $\lambda$ in $B_{X_{n}}(\Lambda)$. Here $X_{n-1}$ is the subalgebra whose Dynkin diagram is obtained from that of $X_{n}$ by removing node 1. There is a natural homomorphism of the weight lattices $\pi: P\left(X_{n}\right) \rightarrow P\left(X_{n-1}\right)$, where $\pi\left(\alpha_{i}^{X_{n}}\right)=\alpha_{i-1}^{X_{n-1}}$ and $\pi\left(\varpi_{i}^{X_{n}}\right)=\varpi_{i-1}^{X_{n-1}}$, and the partition $\lambda$ is identified with the $X_{n-1}$ weights under $\pi$. We identify the Kashiwara operators $\tilde{f}_{i}^{X_{n-1}}$ with $\tilde{f}_{i}^{X_{n}}$ under the embedding.

Explicitly, the bijection $\Phi$ is constructed as follows. Define a string of operators $\tilde{f}_{\overrightarrow{\mathbf{a}}}:=\tilde{f}_{a_{1}} \tilde{f}_{a_{2}} \cdots \tilde{f}_{a_{\ell}}$ such that $\Phi(P)=\tilde{f}_{\overrightarrow{\mathbf{a}}} u$, where $u$ is the highest weight vector in $B_{X_{n}}(\Lambda)$, where $\tilde{f}_{i}$ is the Kashiwara crystal operator corresponding to $f_{i}$. Start with $\overrightarrow{\mathbf{a}}=()$. Scan the columns of $P$ from right to left. For each column of $P$ for which a + can be added, append $(1,2, \ldots, h)$ to $\overrightarrow{\mathbf{a}}$, where $h$ is the height of the added + . Next scan $P$ from left to right and for each column that contains a - in $P$, append to $\overrightarrow{\mathbf{a}}$ the string $(1,2, \ldots, n, n-2, n-3, \ldots, h)$ for type $D_{n}$, $(1,2, \ldots, n-1, n, n, n-1, \ldots, h)$ for type $B_{n}$, and $(1,2, \ldots, n-1, n, n-1, \ldots, h)$ for type $C_{n}$, where $h$ is the height of the - in $P$. Note that for type $C_{n}$ the strings $(1,2, \ldots, h)$ and $(1,2, \ldots, n-1, n, n-1, \ldots, h)$ are the same for $h=n$, which is why empty columns of height $n$ are excluded for \pm diagrams of type $A_{2 n-1}^{(2)}$.

By construction the automorphism $\sigma$ commutes with $\tilde{f}_{i}$ and $\tilde{e}_{i}$ for $i=2,3, \ldots, n$. Hence it suffices to define $\sigma$ on $X_{n-1}$ highest weight elements. Because of the bijection $\Phi$ between \pm diagrams and $X_{n-1}$-highest weight elements, it suffices to define the map on \pm diagrams.

Let $P$ be a \pm diagram of shape $\Lambda / \lambda$. Let $c_{i}=c_{i}(\lambda)$ be the number of columns of height $i$ in $\lambda$ for all $1 \leq i<r$ with $c_{0}=s-\lambda_{1}$. If $i \equiv r-1(\bmod 2)$, then in $P$, above each column of $\lambda$ of height $i$, there must be a + or $\mathrm{a}-$. Interchange the number of such + and - symbols. If $i \equiv r(\bmod 2)$, then in $P$, above each column of $\lambda$ of height $i$, either there are no signs or a $\mp$ pair. Suppose there are $p_{i} \mp$ pairs above the columns of height $i$. Change this to $\left(c_{i}-p_{i}\right) \mp$ pairs. The result is $\mathfrak{S}(P)$, which has the same inner shape $\lambda$ as $P$ but a possibly different outer shape. 
Definition 5.1. Let $b \in \tilde{B}^{r, s}$ and $\tilde{e}_{\overrightarrow{\mathbf{a}}}:=\tilde{e}_{a_{1}} \tilde{e}_{a_{2}} \cdots \tilde{e}_{a_{\ell}}$ be such that $\tilde{e}_{\overrightarrow{\mathbf{a}}}(b)$ is a $X_{n-1}$ highest weight crystal element. Define $\tilde{f}_{\overleftarrow{\mathrm{a}}}:=\tilde{f}_{a_{\ell}} \tilde{f}_{a_{\ell-1}} \cdots \tilde{f}_{a_{1}}$. Then

$$
\sigma(b):=\tilde{f}_{\overleftarrow{\mathbf{a}}} \circ \Phi \circ \mathfrak{S} \circ \Phi^{-1} \circ \tilde{e}_{\overrightarrow{\mathbf{a}}}(b) .
$$

It was shown in 30 , that $\tilde{B}^{r, s}$ is regular.

5.4. Properties of $\tilde{B}^{r, s}$. For the proof of uniqueness we will require the action of $\tilde{e}_{1}$ on $X_{n-2}$ highest weight elements, where $X_{n-2}$ is the Dynkin diagram obtained by removing nodes 1 and 2 from $X_{n}$. As we have seen in section 5.3 , the $X_{n-1}$-highest weight elements in the branching $X_{n} \rightarrow X_{n-1}$ can be described by \pm diagrams. Similarly the $X_{n-2}$-highest weight elements in the branching $X_{n-1} \rightarrow X_{n-2}$ can be described by \pm diagrams. Hence each $X_{n-2}$-highest weight vector is uniquely determined by a pair of \pm diagrams $(P, p)$ such that inner $(P)=\operatorname{outer}(p)$. The diagram $P$ specifies the $X_{n-1}$-component $B_{X_{n-1}}(\operatorname{inner}(P))$ in $B_{X_{n}}(\operatorname{outer}(P))$, and $p$ specifies the $X_{n-2}$ component inside $B_{X_{n-1}}($ inner $(P))$. Let $\Upsilon$ denote the map $(P, p) \mapsto b$ from a pair of \pm diagrams to a $X_{n-2}$ highest weight vector.

To describe the action of $\tilde{e}_{1}$ on an $X_{n-2}$ highest weight element or by $\Upsilon$ equivalently on $(P, p)$ perform the following algorithm:

(1) Successively run through all + in $p$ from left to right and, if possible, pair it with the leftmost yet unpaired + in $P$ weakly to the left of it.

(2) Successively run through all - in $p$ from left to right and, if possible, pair it with the rightmost yet unpaired - in $P$ weakly to the left.

(3) Successively run through all yet unpaired + in $p$ from left to right and, if possible, pair it with the leftmost yet unpaired - in $p$.

Lemma 5.1 (30, Lemma 5.1]). If there is an unpaired + in $p$, $\tilde{e}_{1}$ moves the rightmost unpaired + in $p$ to $P$. Otherwise, if there is an unpaired - in $P, \tilde{e}_{1}$ moves the leftmost unpaired - in $P$ to $p$. Otherwise, $\tilde{e}_{1}$ annihilates $(P, p)$.

In this paper, we will only require the case of Lemma 5.1 when a - from $P$ moves to $p$. Schematically, if a - from a $\mp$ pair in $P$ moves to $p$, then the following happens:

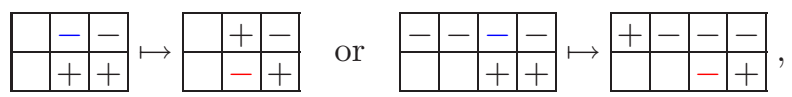

where the blue minus is the minus in $P$ that is being moved and the red minus is the new minus in $p$. Similarly, schematically if a - not part of a $\mp$ pair in $P$ moves to $p$, then

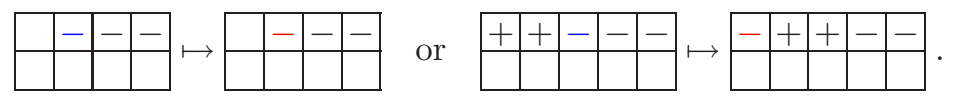

For any $b \in \tilde{B}^{r, s}$, let inner(b) be the inner shape of the \pm diagram corresponding to the $X_{n-1}$ highest weight element in the component of $b$. Furthermore, recall that $\tilde{B}^{r, s}$ is regular, so that in particular $\tilde{e}_{0}$ and $\tilde{e}_{1}$ commute. We can now state the lemma needed in the next section.

Lemma 5.2. Let $b \in \tilde{B}^{r, s}$ be an $X_{n-2}$ highest weight vector corresponding under $\Upsilon$ to the tuple of \pm diagrams $(P, p)$ where inner $(p)=\operatorname{outer}(p)$. Assume that $\varepsilon_{0}(b), \varepsilon_{1}(b)>0$. Then inner $(b)$ is strictly contained in inner $\left(\tilde{e}_{0}(b)\right)$, inner $\left(\tilde{e}_{1}(b)\right)$, and inner $\left(\tilde{e}_{0} \tilde{e}_{1}(b)\right)$. 
Proof. By assumption $p$ does not contain any - and $\tilde{e}_{1}$ is defined. Hence $\tilde{e}_{1}$ moves a - in $P$ to $p$. This implies that the inner shape of $b$ is strictly contained in the inner shape of $\tilde{e}_{1}(b)$.

The involution $\sigma$ does not change the inner shape of $b$ (only the outer shape). By the same arguments as before, the inner shape of $b$ is strictly contained in the inner shape of $\tilde{e}_{1} \sigma(b)$. Since $\sigma$ does not change the inner shape, this is still true for $\tilde{e}_{0}(b)=\sigma \tilde{e}_{1} \sigma(b)$.

Now let us consider $\tilde{e}_{0} \tilde{e}_{1}(b)$. For the change in inner shape we only need to consider $\tilde{e}_{1} \sigma \tilde{e}_{1}(b)$, since the last $\sigma$ does not change the inner shape. By the same arguments as before, $\tilde{e}_{1}$ moves a - from $P$ to $p$ and $\sigma$ does not change the inner shape. The next $\tilde{e}_{1}$ will move another - in $\sigma \tilde{e}_{1}(b)$ to $p$. Hence $p$ will have grown by two - , so that the inner shape of $\tilde{e}_{1} \sigma \tilde{e}_{1}(b)$ is increased by two boxes.

\section{Equivalence of $B^{r, s}$ And $\tilde{B}^{r, s}$ OF Type $D_{n}^{(1)}, B_{n}^{(1)}$, AND $A_{2 n-1}^{(2)}$}

In this section all crystals are of type $D_{n}^{(1)}, B_{n}^{(1)}$, or $A_{2 n-1}^{(2)}$ with corresponding classical subalgebra of type $X_{n}=D_{n}, B_{n}, C_{n}$, respectively.

Let $B$ and $B^{\prime}$ be regular crystals of type $D_{n}^{(1)}, B_{n}^{(1)}$, or $A_{2 n-1}^{(2)}$ with index set $I=\{0,1,2, \ldots, n\}$. We say that $B \simeq B^{\prime}$ is an isomorphism of $J$-crystals if $B$ and $B^{\prime}$ agree as sets and all arrows colored $i \in J$ are the same.

Proposition 6.1. Suppose that there exist two isomorphisms:

$$
\begin{aligned}
& \Psi_{0}: \tilde{B}^{r, s} \simeq B \quad \text { as an isomorphism of }\{1,2, \ldots, n\} \text {-crystals, } \\
& \Psi_{1}: \tilde{B}^{r, s} \simeq B \quad \text { as an isomorphism of }\{0,2, \ldots, n\} \text {-crystals. }
\end{aligned}
$$

Then $\Psi_{0}(b)=\Psi_{1}(b)$ for all $b \in \tilde{B}^{r, s}$ and hence there exists an I-crystal isomorphism $\Psi: \tilde{B}^{r, s} \simeq B$.

Remark 6.1. Note that $\Psi_{0}$ and $\Psi_{1}$ preserve weights, that is, $\operatorname{wt}(b)=\operatorname{wt}\left(\Psi_{0}(b)\right)=$ wt $\left(\Psi_{1}(b)\right)$ for all $b \in \tilde{B}^{r, s}$. This is due to the fact that if all but one coefficient $m_{j}$ are known for a weight $\Lambda=\sum_{j=0}^{n} m_{j} \Lambda_{j}$, then the missing $m_{j}$ is also determined by the level 0 condition.

Proof. If $\Psi_{0}(b)=\Psi_{1}(b)$ for a $b$ in a given $X_{n-1}$-component $C$, then $\Psi_{0}\left(b^{\prime}\right)=\Psi_{1}\left(b^{\prime}\right)$ for all $b^{\prime} \in C$ since $\tilde{e}_{i} \Psi_{0}\left(b^{\prime}\right)=\Psi_{0}\left(\tilde{e}_{i} b^{\prime}\right)$ and $\tilde{e}_{i} \Psi_{1}\left(b^{\prime}\right)=\Psi_{1}\left(\tilde{e}_{i} b^{\prime}\right)$ for $i \in J=$ $\{2,3, \ldots, n\}$. Hence it suffices to prove $\Psi_{0}(b)=\Psi_{1}(b)$ for only one element $b$ in each $X_{n-1}$-component $C$. We are going to establish the theorem for $b$ corresponding to the pairs of \pm diagrams $(P, p)$ where $\operatorname{inner}(p)=\operatorname{outer}(p)$. Note that this is an $X_{n-2}$-highest weight vector, but not necessarily an $X_{n-1}$-highest weight vector.

We proceed by induction on inner $(b)$ by containment. First suppose that both $\varepsilon_{0}(b), \varepsilon_{1}(b)>0$. By Lemma [5.2, the inner shape of $\tilde{e}_{0} \tilde{e}_{1} b, \tilde{e}_{0} b$, and $\tilde{e}_{1} b$ is bigger than the inner shape of $b$, so that by induction hypothesis $\Psi_{0}\left(\tilde{e}_{0} \tilde{e}_{1} b\right)=\Psi_{1}\left(\tilde{e}_{0} \tilde{e}_{1} b\right)$, $\Psi_{0}\left(\tilde{e}_{0} b\right)=\Psi_{1}\left(\tilde{e}_{0} b\right)$, and $\Psi_{0}\left(\tilde{e}_{1} b\right)=\Psi_{1}\left(\tilde{e}_{1} b\right)$. Therefore we obtain

$$
\begin{aligned}
\tilde{e}_{0} \tilde{e}_{1} \Psi_{0}(b)=\tilde{e}_{0} \Psi_{0}\left(\tilde{e}_{1} b\right)=\tilde{e}_{0} \Psi_{1}\left(\tilde{e}_{1} b\right)= & \Psi_{1}\left(\tilde{e}_{0} \tilde{e}_{1} b\right)=\Psi_{0}\left(\tilde{e}_{0} \tilde{e}_{1} b\right) \\
& =\tilde{e}_{1} \Psi_{0}\left(\tilde{e}_{0} b\right)=\tilde{e}_{1} \Psi_{1}\left(\tilde{e}_{0} b\right)=\tilde{e}_{1} \tilde{e}_{0} \Psi_{1}(b) .
\end{aligned}
$$

This implies that $\Psi_{0}(b)=\Psi_{1}(b)$.

Next we need to consider the cases when $\varepsilon_{0}(b)=0$ or $\varepsilon_{1}(b)=0$, which comprises the base case of the induction. Let us first treat the case $\varepsilon_{1}(b)=0$. Recall that 
inner $(p)=\operatorname{outer}(p)$ so that $p$ contains only empty columns. Hence it follows from the description of the action of $\tilde{e}_{1}$ of Lemma 5.1 that $\varepsilon_{1}(b)=0$ if and only if $P$ consists only of empty columns or columns containing + .

Claim. $\Psi_{0}(b)=\Psi_{1}(b)$ for all $b$ corresponding to the pair of \pm diagrams $(P, p)$ where $P$ contains only empty columns and columns with + , and $\operatorname{inner}(p)=\operatorname{outer}(p)$.

The claim is proved by induction on $k$, which is defined to be the number of empty columns in $P$ of height strictly smaller than $r$. For $k=0$ the claim is true by weight considerations. Now assume the claim is true for all $0 \leq k^{\prime}<k$ and we will establish the claim for $k$. Suppose that $\Psi_{1}(b)=\Psi_{0}(\tilde{b})$ where $\tilde{b} \neq b$. By weight considerations $\tilde{b}$ must correspond to a pair of \pm diagrams $(\tilde{P}, p)$, where $\tilde{P}$ has the same columns containing + as $P$, but some of the empty columns of $P$ of height $h$ strictly smaller than $r$ could be replaced by columns of height $h+2$ containing $\mp$. Denote by $k_{+}$the number of columns of $P$ containing + . Then

$$
m:=\varepsilon_{0}(b)=k_{+}+k,
$$

since under $\sigma$ all empty columns in $P$ become columns with \pm and columns containing + become columns with -. By Lemma 5.1, then $\tilde{e}_{1}$ acts on $(\mathfrak{S}(P), p)$ as often as there are minus signs in $\mathfrak{S}(P)$, which is $k_{+}+k$. Set $\hat{b}=\tilde{e}_{1}^{a} \tilde{b}$, where $a>0$ is the number of columns in $\tilde{P}$ containing $\mp$. If $(\hat{P}, \hat{p})$ denotes the tuple of \pm diagrams associated to $\hat{b}$, then compared to $(\tilde{P}, p)$ all - from the $\mp$ pairs in $\tilde{P}$ moved to $p$. Note that $\hat{P}$ has only $k-a<k$ empty columns of height less than $r$, so that by induction hypothesis $\Psi_{0}(\hat{b})=\Psi_{1}(\hat{b})$. Hence

$$
\Psi_{1}(b)=\Psi_{0}(\tilde{b})=\Psi_{0}\left(\tilde{f}_{1}^{a} \hat{b}\right)=\tilde{f}_{1}^{a} \Psi_{0}(\hat{b})=\tilde{f}_{1}^{a} \Psi_{1}(\hat{b}) .
$$

Note that

$$
\varepsilon_{0}(\hat{b})=\varepsilon_{0}(\tilde{b})=m-a<m
$$

Hence

$$
\tilde{e}_{0}^{m} \Psi_{1}(b)=\Psi_{1}\left(\tilde{e}_{0}^{m} b\right) \neq 0,
$$

but

$$
\tilde{e}_{0}^{m} \tilde{f}_{1}^{a} \Psi_{1}(\hat{b})=\tilde{f}_{1}^{a} \Psi_{1}\left(\tilde{e}_{0}^{m} \hat{b}\right)=0
$$

which contradicts (6.1). This implies that we must have $\tilde{b}=b$ proving the claim.

The case $\varepsilon_{0}(b)=0$ can be proven in a similar fashion to the case $\varepsilon_{1}(b)=0$. Using the explicit action of $\mathfrak{S}$ on $P$ and Lemma 5.1, it follows that $\varepsilon_{0}(b)=0$ if and only if $P$ consists only of columns containing - or $\mp$ pairs.

Claim. $\Psi_{0}(b)=\Psi_{1}(b)$ for all $b$ corresponding to the pair of \pm diagrams $(P, p)$ where $P$ contains only columns with - and columns with $\mp$ pairs, and inner $(p)=\operatorname{outer}(p)$.

By induction on the number of $\mp$ pairs in $P$, this claim can be proven similarly as before (using the fact that $\mathfrak{S}$ changes columns with - into columns with + and columns with $\mp$ pairs into empty columns).

Proof of Theorem 1.2, Both crystals $B^{r, s}$ and $\tilde{B}^{r, s}$ have the same classical decomposition (5.1) as $X_{n}$ crystals with index set $\{1,2, \ldots, n\}$ and $\{0,2,3, \ldots, n\}$ by 
Proposition 4.2. Hence there exist crystal isomorphisms $\Psi_{0}$ and $\Psi_{1}$. By Proposition 6.1 there exists an $I$-isomorphism $\Psi: \tilde{B}^{r, s} \cong B^{r, s}$ which proves the theorem.

\section{ACKNOWLEDGMENTS}

The first author thanks Masaki Kashiwara for letting him know that the irreducibility of $W_{s}^{(r)}$ in Proposition 3.6 follows from his result in [17, and Hiraku Nakajima for calling his attention to references on the polarization of $V\left(\varpi_{r}\right)$. The authors would also like to thank David Hernandez for helpful correspondences. M.O. is partially supported by Grant-in-Aid for Scientific Research (C) 18540030, Japan Society for the Promotion of Science. A.S. is partially supported by NSF grants DMS-0501101, DMS-0652641, and DMS-0652652.

\section{REFERENCES}

[1] J. Beck and H. Nakajima, Crystal bases and two-sided cells of quantum affine algebras, Duke Math. J. 123 (2004), no. 2, 335-402. MR2066942(2005e:17020)

[2] G. Benkart, I. Frenkel, S-J. Kang, and H. Lee, Level 1 perfect crystals and path realizations of basic representations at $q=0$, Int. Math. Res. Not. 2006, Art. ID 10312, 28 pp. MR2272099 (2007i:17030)

[3] V. Chari, On the fermionic formula and the Kirillov-Reshetikhin conjecture, Internat. Math. Res. Notices 12 (2001) 629-654. MR1836791 (2002i:17019)

[4] V. Chari and A. Pressley, Quantum affine algebras and their representations, in Representations of groups, CMS Conf. Proc. 16, Amer. Math. Soc., Providence, RI (1995) 59-78. MR:1357195 (96j:17009)

[5] V. Chari, A. Pressley, Twisted quantum affine algebras, Comm. Math. Phys. 196 (1998) 461-476 MR1645027 (99f:16041)

[6] P. Di Francesco, R. Kedem, Proof of the combinatorial Kirillov-Reshetikhin conjecture, preprint arXiv:0710.4415.

[7] G. Hatayama, A. Kuniba, M. Okado, T. Takagi, and Z. Tsuboi, Paths, crystals and fermionic formulae, MathPhys Odyssey 2001, 205-272, Prog. Math. Phys. 23, Birkhäuser Boston, Boston, MA, 2002. MR 1903978 (2003e:17020)

[8] G. Hatayama, A. Kuniba, M. Okado, T. Takagi, and Y. Yamada, Remarks on fermionic formula, Contemporary Math. 248 (1999) 243-291. MR1745263 (2001m:81129)

[9] D. Hernandez, The Kirillov-Reshetikhin conjecture and solution of T-systems, J. Reine Angew. Math. 596 (2006) 63-87. MR 2254805 (2007j:17020)

[10] D. Hernandez, Kirillov-Reshetikhin conjecture: The general case, preprint arXiv:0704.2838.

[11] J. Hong and S.-J. Kang, Introduction to Quantum Groups and Crystal Bases, Graduate Studies in Mathematics, Volume 42, American Mathematical Society, 2002. MR 1881971 (2002m:17012)

[12] V. G. Kac, Infinite Dimensional Lie Algebras, 3rd ed., Cambridge Univ. Press, Cambridge, UK, 1990. MR 1104219 (92k:17038)

[13] S-J. Kang, M. Kashiwara, K. C. Misra, T. Miwa, T. Nakashima and A. Nakayashiki, Affine crystals and vertex models, Int. J. Mod. Phys. A 7 (suppl. 1A) (1992), 449-484. MR1187560 (94a:17008)

[14] S-J. Kang, M. Kashiwara, K. C. Misra, T. Miwa, T. Nakashima and A. Nakayashiki, Perfect crystals of quantum affine Lie algebras, Duke Math. J. 68 (1992) 499-607. MR.1194953 (94j:17013)

[15] M. Kashiwara, On crystal bases of the q-analogue of universal enveloping algebras, Duke Math. J. 63 (1991), 465-516. MR1115118 (93b:17045)

[16] M. Kashiwara, Crystal bases of modified quantized enveloping algebra, Duke Math. J. 73 (1994), 383-413. MR:1262212(95c:17024)

[17] M. Kashiwara, On level zero representations of quantized affine algebras, Duke Math. J. 112 (2002) 117-175. MR 1890649 (2002m:17013)

[18] M. Kashiwara and T. Nakashima, Crystal graphs for representations of the q-analogue of classical Lie algebras, J. Alg. 165 (1994) 295-345. MR1273277 (95c:17025) 
[19] Y. Koga, Level one perfect crystals for $B_{n}^{(1)}, C_{n}^{(1)}$, and $D_{n}^{(1)}$, J. Algebra 217 (1999), no. 1, 312-334. MR 1700489(2000h:17011)

[20] A. Kuniba and T. Nakanishi, The Bethe equation at $q=0$, the Möbius inversion formula, and weight multiplicities II. The $X_{n}$ case, J. Algebra 251 (2002) 577-618. MR1917385 (2003m:17013)

[21] A. Kuniba, T. Nakanishi and Z. Tsuboi, The canonical solutions of the Q-systems and the Kirillov-Reshetikhin conjecture, Commun. Math. Phys. 227 (2002) 155-190. MR1903843 (2003e:81085)

[22] G. Lusztig, Introduction to quantum groups, Progress in Math. 110, Birkhäuser, 1993. MR:1227098 (94m:17016)

[23] H. Nakajima, Extremal weight modules of quantum affine algebras, Representation theory of algebraic groups and quantum groups, 343-369, Adv. Stud. Pure Math., 40, Math. Soc. Japan, Tokyo, 2004. MR2074599 (2005g:17036)

[24] H. Nakajima, $t$-analogues of q-characters of Kirillov-Reshetikhin modules of quantum affine algebras, Represent. Theory 7 (2003) 259-274. MR:1993360 (2004e:17013)

[25] H. Nakajima, t-analogues of q-characters of quantum affine algebras of type $E_{6}, E_{7}, E_{8}$, preprint arXiv:math.QA/0606637.

[26] S. Naito and D. Sagaki, Construction of perfect crystals conjecturally corresponding to Kirillov-Reshetikhin modules over twisted quantum affine algebras, Comm. Math. Phys. 263 (2006), no. 3, 749-787. MR2211823 (2007a:17023)

[27] M. Okado, Existence of Crystal Bases for Kirillov-Reshetikhin Modules of Type D, Publ. RIMS 43 (2007), 977-1004.

[28] M. Okado, A. Schilling, and M. Shimozono, Virtual crystals and fermionic formulas of type $D_{n+1}^{(2)}, A_{2 n}^{(2)}$, and $C_{n}^{(1)}$, Representation Theory 7 (2003) 101-163. MR 1973369(2004f:17023)

[29] M. Okado, A. Schilling, and M. Shimozono, Virtual crystals and Kleber's algorithm, Commun. Math. Phys. 238 (2003) 187-209. MR1989674 (2004c:17034)

[30] A. Schilling, The combinatorial structure of Kirillov-Reshetikhin crystals of type $D_{n}^{(1)}, B_{n}^{(1)}$, $A_{2 n-1}^{(2)}$, J. Algebra, 319 (2008), 2938-2962.

[31] M. Shimozono, Affine type A crystal structure on tensor products of rectangles, Demazure characters, and nilpotent varieties, J. Algebraic Combin. 15 (2002), no. 2, 151-187. MR1887233 (2002m:17005)

[32] P. Sternberg, Applications of crystal bases to current problems in representation theory, Ph.D. thesis, UC Davis 2006 (available at arXiv:math.QA/0610704).

[33] M. Varagnolo and E. Vasserot, Canonical bases and quiver varieties, Represent. Theory 7 (2003), 227-258. MR1990661 (2004i:17012)

[34] S. Yamane, Perfect crystals of $U_{q}\left(G_{2}^{(1)}\right)$, J. Algebra 210 (1998), no. 2, 440-486. MR1662347 (2000f:17024)

Department of Mathematical Science, Graduate School of Engineering Science, OSAKa University, TOYONAKA, OSAKa 560-8531, JAPAN

E-mail address: okado@sigmath.es.osaka-u.ac.jp

Department of Mathematics, University of California, One Shields Avenue, Davis, CALifornia 95616-8633

E-mail address: anne@math.ucdavis.edu

$U R L:$ http://www.math.ucdavis.edu/ anne 